\title{
Advances in nonlinear optical microscopy techniques for in vivo and in vitro neuroimaging
}

\author{
Sparsha Pallen ${ }^{1} \cdot$ Yuthika Shetty ${ }^{1} \cdot$ Subir Das ${ }^{2} \cdot$ Joel Markus Vaz $^{3} \cdot$ Nirmal Mazumder $^{1}$ (I)
}

Received: 3 June 2021 / Accepted: 17 August 2021 / Published online: 31 August 2021

(C) The Author(s) 2021

\begin{abstract}
Understanding the mechanism of the brain via optical microscopy is one of the challenges in neuroimaging, considering the complex structures. Advanced neuroimaging techniques provide a more comprehensive insight into patho-mechanisms of brain disorders, which is useful in the early diagnosis of the pathological and physiological changes associated with various neurodegenerative diseases. Recent advances in optical microscopy techniques have evolved powerful tools to overcome scattering of light and provide improved in vivo neuroimaging with sub-cellular resolution, endogenous contrast specificity, pinhole less optical sectioning capability, high penetration depth, and so on. The following article reviews the developments in various optical imaging techniques including two-photon and three-photon fluorescence, second-harmonic generation, third-harmonic generation, coherent anti-Stokes Raman scattering, and stimulated Raman scattering in neuroimaging. We have outlined the potentials and drawbacks of these techniques and their possible applications in the investigation of neurodegenerative diseases.
\end{abstract}

Keywords Optical microscopy $\cdot$ Multiphoton fluorescence microscopy $\cdot$ Harmonic generation $\cdot$ Coherent Raman scattering Neuroimaging

\section{Introduction}

Neurodegenerative disorders (ND) affect as much as $30 \%$ of the elderly population over 85 years of age. An estimated 46 million people worldwide have been diagnosed with ND such as Alzheimer's disease (AD) and other dementia, and is expected to surpass 135 million by 2050 (Lassonde et al. 2017). Alzheimer's disease, amyotrophic lateral sclerosis (ALS), Huntington disease (HD), multiple sclerosis (MS), Parkinson's disease (PD), and transmissible spongiform encephalitis (TSE) are a few of the ND characterized by progressive damage of the neurons leading to cognitive impairment

Nirmal Mazumder

nirmaluva@gmail.com

1 Department of Biophysics, Manipal School of Life Sciences, Manipal Academy of Higher Education,

Manipal, Karnataka 576104, India

2 Institute of Biophotonics, National Yang Ming Chiao Tung University, No. 155, Sec. 2, Linong St., Taipei 112, Taiwan

3 Department of Biotechnology, Manipal Institute of Technology, Manipal, Karnataka 576104, India and motor dysfunctions (Soto 2003; Weller and Budson 2018). In vivo studies are performed to analyze the brain in real time. Conventional functional visualization neuroimaging techniques such as magnetic resonance imaging (MRI), positron emission tomography (PET), and computerized tomography $(\mathrm{CT})$ are currently used for imaging the whole brain. These techniques are expensive and provide poor spatial and temporal resolution (Ross and Poirier 2004). Diverse structural optical imaging techniques such as laser scanning confocal microscopy and widefield microscopy are useful for detecting neurodegeneration. However, the challenges include the optimization of linear optical parameters for deep brain imaging by them. Prolonged exposure of the sample to lasers and fluorophores can induce deleterious effects like phototoxicity and photobleaching (St. Croix et al. 2005). Nonlinear optical (NLO) techniques utilize the difference in optical attenuation and cellular complexity of tissue samples to achieve excellent imaging sensitivity. NLO microscopy techniques such as two photon fluorescence (2PF) and three-photon fluorescence (3PF) microscopy can substantially reduce photo-bleaching, photo-toxicity, minimize signal loss, and used for analyzing the neuronal structure and functions (Denk et al. 1990; Xu et al. 1996; Campagnola 2011; Squier et al. 1998; Zumbusch et al. 1999; Nandakumar et al. 2009). Second harmonic 
generation (SHG) and third harmonic generation (THG) microscopy allow improved visualization of the cellular morphology of the brain tissue samples with high specificity and sensitivity. THG microscopy can be used to estimate lipid compositions, one of the parameters for identifying the progression of ND. They are also used to image the nucleus, plasma membrane, and membranebased organelles such as mitochondria. Studies have shown that $3 \mathrm{PF}$ and THG microscopy can reveal information on melanin content in the skin, outspreading their applications to the field of dermatology (Sun et al. 2019). Coherent anti-Stokes Raman scattering (CARS) and stimulated Raman scattering (SRS) microscopy are beginning to gain attention as a promising, label-free chemical imaging technique for sensitive 3D visualization of tissue architecture. As mentioned above, all the NLO techniques fulfill the fundamental requirements essential for obtaining 3D images with high resolution and elevated signal-to-noise ratio. With active transformations in optics, NLO techniques can enhance the applications of brain imaging to study disease progression in ND. In this review, we discuss the potential of optical techniques, including 2PF, 3PF, SHG, THG, CARS, SRS, and other techniques that have gained importance in the field of neuroimaging.

\section{Experimental setup}

The 2PF microscope uses an ultrashort pulse of near-infrared (NIR) wavelength for two-photon excitation. In 2PF, the excitation wavelength is chosen such that the fluorophore absorbs two photons simultaneously and emit a fluorescence signal with lower energy. Fig 1 (A) illustrates the schematic of a $2 \mathrm{PF}$ microscopy setup. In 3PF microscopy, three photons are simultaneously absorbed by a fluorophore from a highdensity NIR laser beam. Fig 1 (B) illustrates the schematic of a 3PF microscopy setup with an axicon-based Bessel beam (Rodríguez et al. 2018). SHG is a second-order nonlinear scattering phenomenon that is used to image noncentrosymmetric molecules (Mazumder et al. 2014). It occurs when two photons of lower energy from an incident laser source are absorbed by a molecule and emitted as a single photon of precisely double the incident frequency. The resultant SHG intensity depends on the relative orientation between the polarization of the incoming light and the second-order hyperpolarizability of the sample. The specimens are subjected to intense, focused, short pulses of radiation of longer wavelengths (near IR region) to enable higher tissue penetration. Since the SH signal obtained depends on the polarity of molecules, labeling can be avoided in SHG imaging, making it an excellent candidate for in vivo imaging with the least damaging effect. Fig 1 (C) depicts the forward and backward collection experimental setup of the SHG microscopy (Sivaguru et al. 2010). THG is a third-order NLO process where three photons of its fundamental frequency interact with a nonlinear material and then generate a single photon (Squier et al. 1998; Farrar et al. 2011). Fig 1 (D) shows the experimental setup for THG microscopy and the signal is collected in the forward direction using photomultiplier tube (PMT) (Chen et al. 2015). In CARS microscopy, $\omega_{P}$ i.e., pump and $\omega_{S}$ i.e., Stokes beam of different frequencies are illuminated onto the sample simultaneously, and a resultant anti-Stokes signal is generated when the difference in frequencies between the pump and Stokes beam matches with the Raman vibrational frequency of a molecule (Zumbusch et al. 1999; Evans et al. 2005; Cheng et al. 2002). Fig 1 (E) shows the experimental setup of CARS microscopy. E-CARS (Epi or backward detection) detects the signals that arise from samples whose axial length is much smaller than the incident wavelength. In contrast, FCARS (forward detection) detects signals from samples with comparable or larger axial lengths than that of the excitation wavelength. E-CARS improves the image contrast as it exhibits a lesser non-resonant background when compared to F-CARS, which is useful for live-cell imaging. In the SRS approach, two laser beams of different frequencies, $\omega_{P}$, i.e., pump and, $\omega_{S}$, i.e., Stokes beams are illuminated on the sample, such that the frequency difference between the pump and the Stokes matches with the molecular vibrational frequency of a chemical bond in a molecule. As a result, the Stokes beam experiences intensity gain (stimulated Raman gain, SRG), and simultaneously, the pump beam experiences intensity loss (stimulated Raman loss, SRL). Therefore, SRG and SRL do not contribute to the non-resonant background, which is a common issue in CARS microscopy. Fig 1 (F) shows the typical SRS imaging experimental setup (Nandakumar et al. 2009; Evans et al. 2007).

\section{Applications}

\section{Two-photon fluorescence (2PF) microscopy}

Optical microscopy techniques have rapidly evolved since the early 1960s, following the invention of the laser. It was another 30 years when Denk et al. (1990) apprehended the first 2PF laser scanning microscopy in 1990 closely following the invention of a mode-locked laser. A NIR light source is usually the choice for an excitation source as it avoids water absorption, thus, reducing photo-bleaching and photo-toxicity within the optical window, and increasing the penetration depth in thick tissue samples (Helmchen and Denk 2005; Lu et al. 2018; Matsumoto et al. 2015). The ability to image deeper brain regions with the single-cell resolution has refined the establishment of synaptic activity and its functions in the brain 


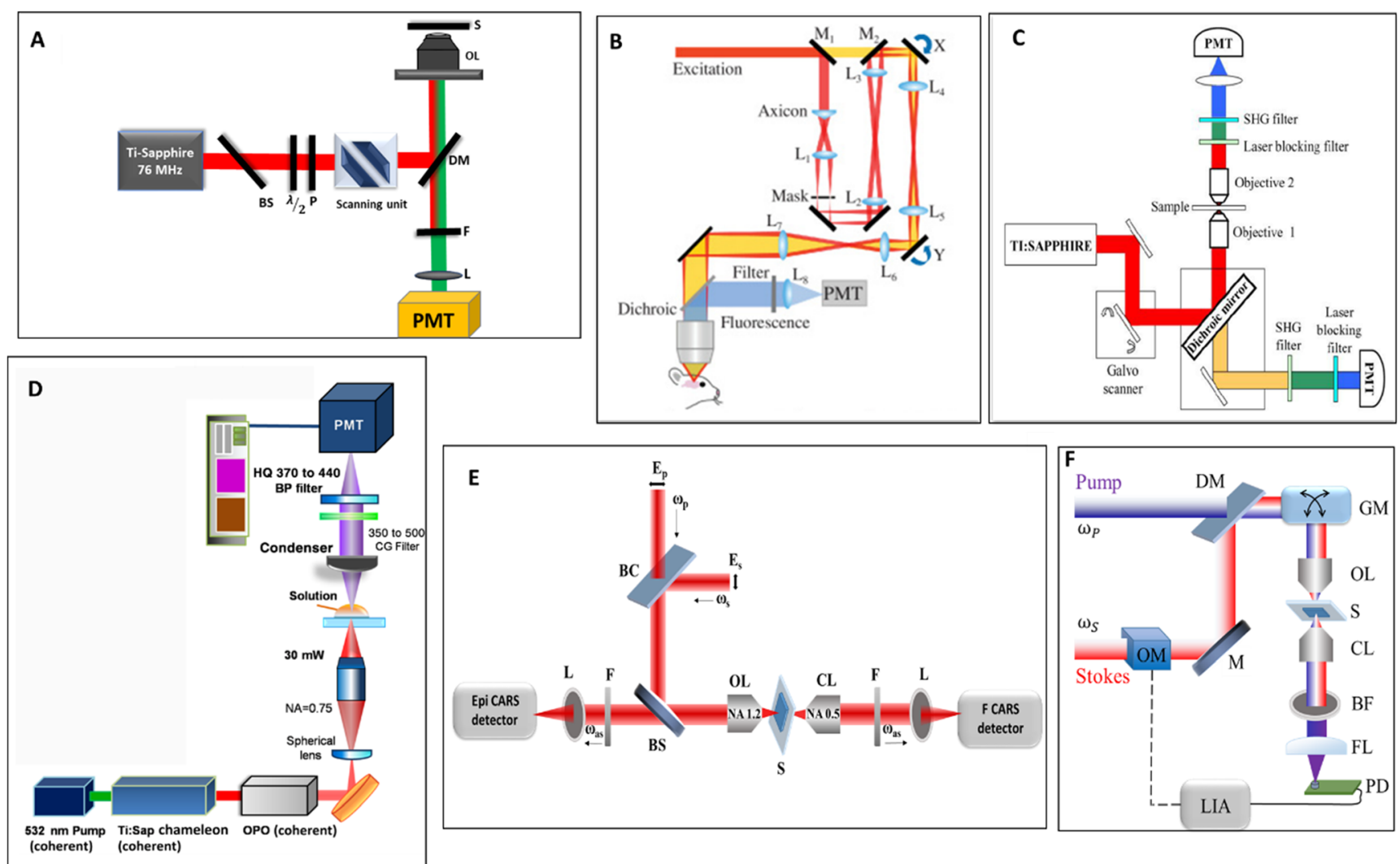

Fig. 1 Schematic experimental setups. (A) Experimental setup of twophoton fluorescence microscope. PD, photo diode; BS, beam splitter; P, polarizer; $\lambda / 2$, half wave-plate; $\lambda / 4$, quarter wave-plate; $\mathrm{DM}$, dichroic mirror; OL, objective lens; S, sample; F, filter; L, lens; PMT, photomultiplier tubes. This figure is adapted with permission from Mittmann et al. 2011. (B) A homebuilt three-photon microscope with an axiconbased Bessel module. M1 and M2 are flip mirrors for switching between Gaussian (yellow path) and Bessel (red path) imaging modalities. L, lenses; X and Y, galvanometers; PMT, photomultiplier tube. This figure is adapted with permission from Rodríguez et al. (2018). (C) Forward and backward collected SHG microscope. The apparatus is built using Zeiss LSM 710 microscope and a tunable Ti:sapphire laser source at

(Matsumoto et al. 2015). In addition to the contributions of $2 \mathrm{PF}$ in exploring the intracellular $\mathrm{Ca}^{2+}$ dynamics, it is also employed in in vivo studies to estimate the electrical activities of brain cells (Mittmann et al. 2011). $\mathrm{Ca}^{2+}$ dynamics is established as one of the forms of communication between neighboring neurons within astrocytes (Araque et al. 2002; Perea and Araque 2005). These studies shed light on the importance of tripartite communication between neuronal and glial cells in network functioning. The mechanism of formation of $\mathrm{A} \beta$ plaques, one of the hallmarks of $\mathrm{AD}$ condition, can be studied using a $2 \mathrm{PF}$ microscope to obtain a real-time, $3 \mathrm{D}$ visualization of the condition. In a study, a quadripolar twophoton probe (QAD1) was developed for in vivo imaging which showed a larger cross-section, high photostability, specificity, and sensitivity for amyloid plaques (Heo et al. 2016). Fig. 2 shows the in vivo $2 \mathrm{PF}$ image of the frontal cortex of transgenic 5XFAD mice at different time points following wavelength $780 \mathrm{~nm}$. This figure is adapted with permission from Sivaguru et al. (2010). (D) Optical arrangement of a THG microscope. This figure is adapted with permission from Chen et al. (2015). (E) Epi and forward detection CARS microscope. BC, beam combiner; BS, beam splitter; F, filter; L, lens; OL, objective lens; S, sample; CL, condenser lens; F, filter. This figure is adapted with permission from Evans et al. (2005). (F) SRS microscope. OM, optical modulator; M, mirror; DM, dichroic mirror; GM, galvo mirror; OL, objective lens; S, sample; CL, condenser lens; BF, bandpass filter; FL, focusing lens; PD, photodiode; LIA, lock-in amplifier. This figure is adapted with permission from Nandakumar et al. (2009)

injection with QAD1. The 3D reconstruction of $2 \mathrm{PF}$ images revealed the accumulation and distribution of $A \beta$ plaques. In vivo $2 \mathrm{PF}$ imaging was used to examine the plaque microenvironment in the AD mice model which displayed abnormal fluctuations in glutamate concentrations. Parenchymal microglia are a major component of the immune system in the brain. Microglia are also activated in the case of any inflammatory effects and neuronal degeneration. It is inhibited by apyrase, G-protein coupled purinergic receptors, and connexin channels, which are present in high amounts in astrocytes, suggesting that they control microglial response to major tissue damage or traumatic injury (Davalos et al. 2005). During major tissue damage events, on-site microglial processing can be visualized using $2 \mathrm{PF}$.

To precisely understand the mechanism of any protein in regulating neuronal activity, scientists heavily rely on conventional genetic inhibition or knockouts. Recently, 2PF 

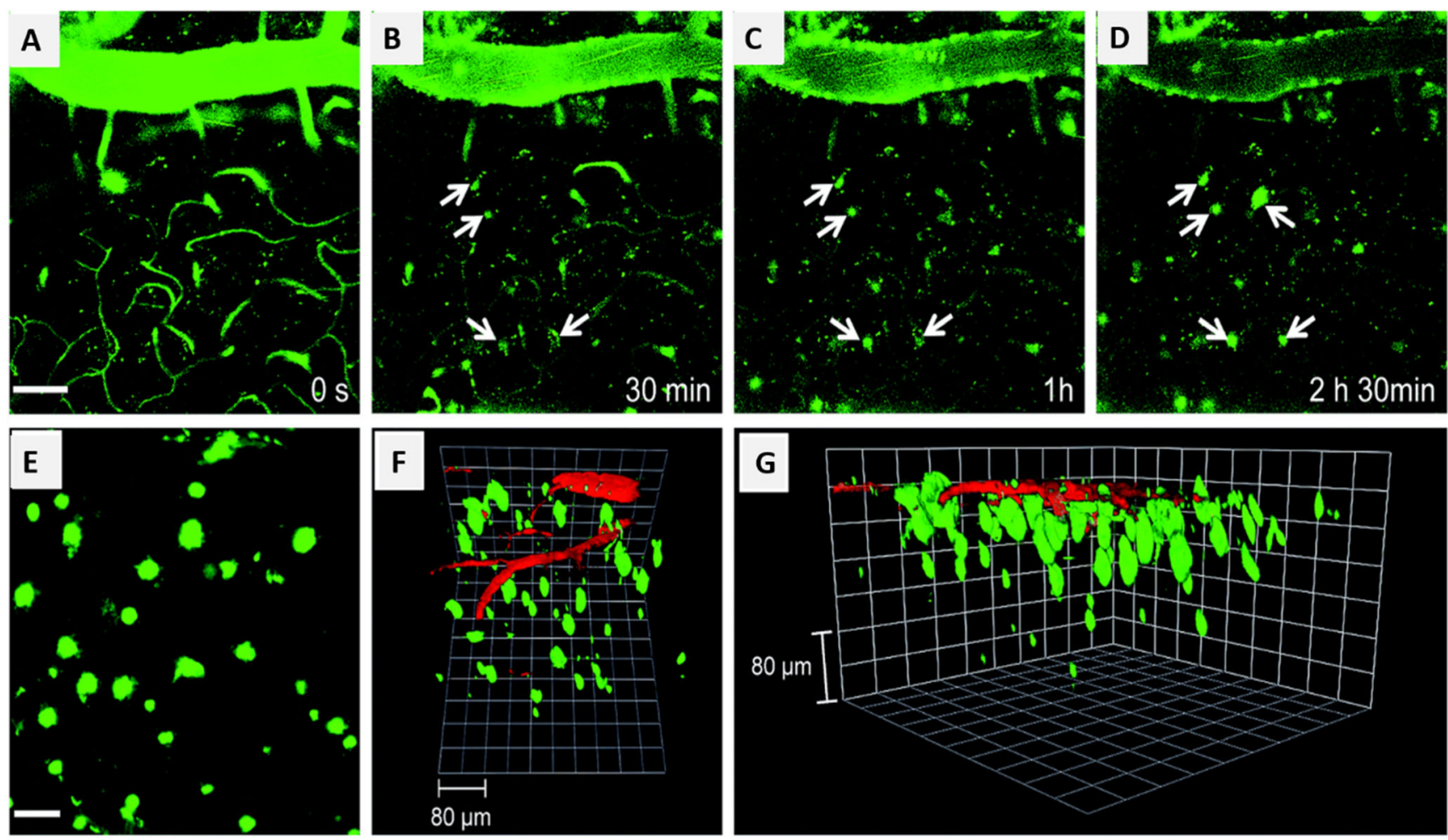

Fig. 2 Transgenic mice frontal cortex in vivo $2 \mathrm{PF}$ images at (A) 0 , (B) 30 , (C) 60 , and (D) $150 \mathrm{~min}$ after i.v. injection of QAD1 $\left(10 \mathrm{mg} \mathrm{kg}^{-1}\right)$, noted the observation of plaques over time. (E) $2 \mathrm{PF}$ images at the depth of $\sim 300 \mu \mathrm{m}$ from the surface of the cortex along the $z$-direction show the

microscopy has allowed reversible activation and deactivation of neuronal receptors in a cell-type specific manner resulting in better biological perturbation in a controlled spatiotemporal resolution. Pittolo et al. (2019) used photo-switchable allosteric modulators (or alloswitch) and $2 \mathrm{PF}$ microscope to selectively silence metabotropic glutamate 5 (mGlu5) receptors in brain tissues to investigate the functional role of the receptor. Thus, 2PF microscopy has advanced to be a valuable tool for the analysis of neuronal structure and functions, such as longterm potentiation, calcium signaling pathway, microglial movement, calcium dysregulation, and synaptic plasticity, which can be exploited to study the onset and development of ND (Kawakami et al. 2015; Sahu and Mazumder 2020). However, this technique is limited due to low penetration capacity in tissue.

\section{Three-photon fluorescence (3PF) microscopy}

The 3PF microscopy is a technique for high-resolution, deep tissue in vivo imaging. Currently, the $3 \mathrm{PF}$ microscope also shows immense potential in tissue diagnostics as it allows the use of a convenient and compact fiber laser as an excitation source, thus reducing expenses and increasing clinical compatibility. Image subcortical structures present at significant depths in an intact mouse brain is possible using $3 \mathrm{PF}$

distribution of $\mathrm{A} \beta$ plaques. $(\mathrm{F})$ and $(\mathrm{G}) 3 \mathrm{D}$ reconstructed $2 \mathrm{PF}$ image of transgenic mice frontal cortex after i.v. injection of QAD1 $\left(10 \mathrm{mg} \mathrm{kg}^{-1}\right)$ and dextran $40 \mathrm{kDa}$ Texas red. Scale bars: $50 \mu \mathrm{m}$ in (A) and $30 \mu \mathrm{m}$ in (E), respectively (Heo et al. 2016)

microscopy. The depth of imaging in $2 \mathrm{PF}$ is limited by tissue scattering and to overcome it, the current solutions are either the removal of brain tissue or insertion of optical probes. 3PF microscopy can capture images at a depth greater than that of 2PF microscopy as it utilizes higher wavelengths, which decreases tissue scattering and attenuation of the excitation wavelength (Horton et al. 2013). The optimal spectral window for the sample is near $1700 \mathrm{~nm}$ taking into consideration tissue scattering and absorption which allows the excitation of several fluorophores such as fluorescent proteins, and calcium indicators (Xu et al. 1996). Intact brain imaging of the mouse hippocampus was successfully performed by acquiring images of neurons labeled with a red fluorescent protein in B6.Cg-Tg (Thy1-Brainbow1.0) HLich/J mouse. The fluorescently labeled neurons were imaged at a depth of 1060-1120 $\mu \mathrm{m}$ below the outer surface of the brain (Horton et al. 2013). $3 \mathrm{PF}$ microscope provides a significant advantage over $2 \mathrm{PF}$ with its longer wavelengths resulting in increased scattering length and reduced out-of-focus background signal. Yildirim et al. (2019) carried out a vertical column imaging of the cerebral cortex in a live mouse by optimizing the parameters of $3 \mathrm{PF}$ to produce an energy-efficient response and limit the damage encountered by the tissue. They investigated the functional visual responses of neurons in the primary visual cortex (layer V1) and white matter below cortical layer VI 
A

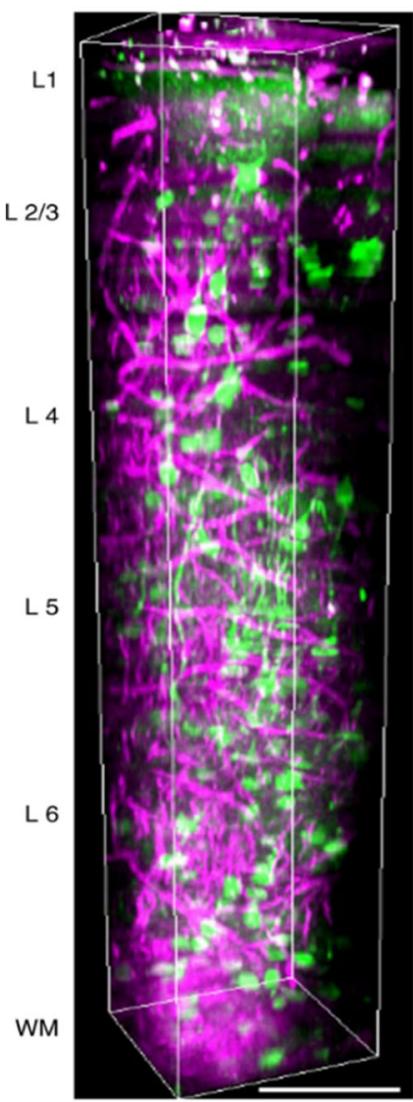

B
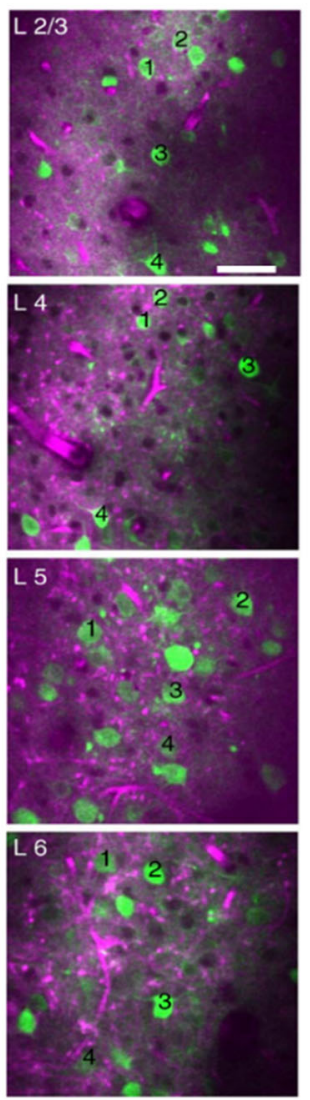

C
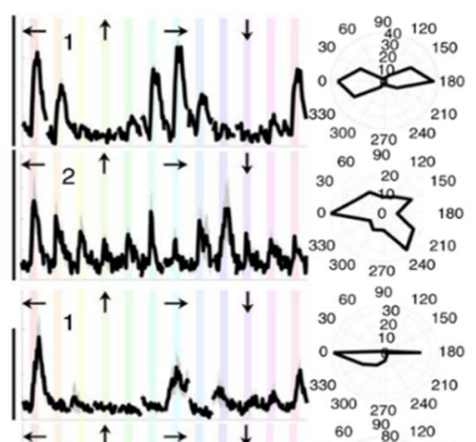

$60 \quad 90 \quad 120$

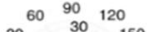

\begin{tabular}{l}
$30 \begin{array}{l}30 \\
20 \\
10\end{array}$ \\
$\quad 180$ \\
\hline
\end{tabular}
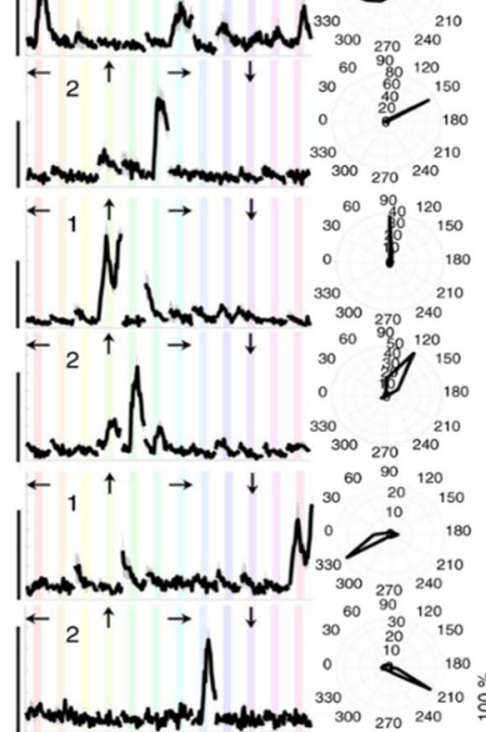
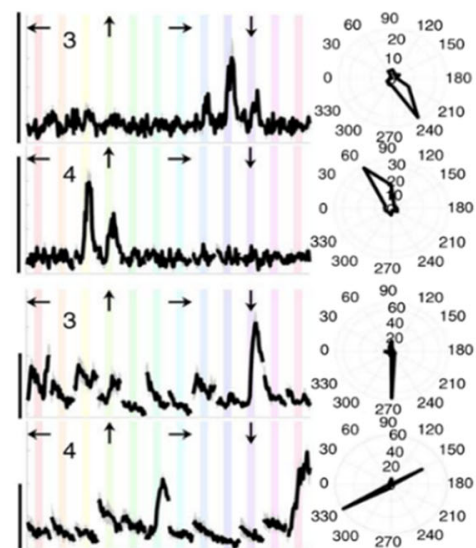

$\rightarrow \rightarrow+\downarrow$
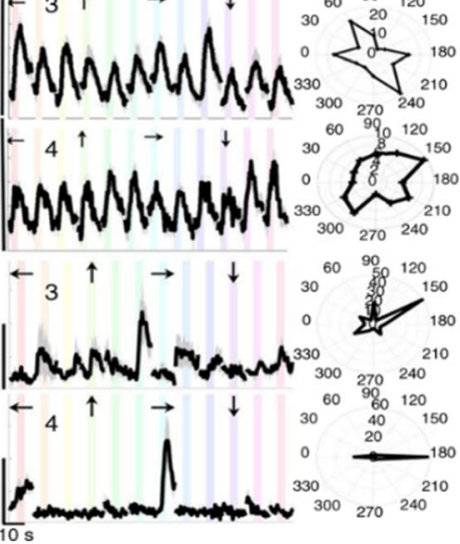

Fig. 3 Visual response characterization at different layers in V1 of awake mice. (A) 3D rendering of a sequence of 450 lateral 3PF images acquired with an increment of $2 \mu \mathrm{m}$. GCaMP6s signal is represented with green color. Scale bars, $100 \mu \mathrm{m}$. (B) Selection of lateral images from layers $2 / 3$, 4, 5, and 6 with $250 \mu \mathrm{m}$ field of view; scale bar, $50 \mu \mathrm{m}$. (C) Average calcium responses $(\Delta F / F)$ of over 10 trials of representative cells in each

in the subplate (as shown in Fig. 3). It was concluded that deep tissue imaging had no disruptions from the superficial layers of neurons. Structural imaging of the entire cortex and white matter revealed that neurons and blood vessels connected to these regions were present below the cortex. $3 \mathrm{PF}$ imaging can be used to identify blood vessels, as well as myelinated axons, and reveal the functional visual response of excitatory neurons in layer VI expressed with GCaMP6s in mice.

GCaMP6 indicators respond to the changes in $\mathrm{Ca}^{2+}$ concentrations; their neutral (GCaMP6 bound to $\mathrm{Ca}^{2+}$ ) and anionic forms have different fluorescence excitation spectra (Ouzounov et al. 2019). Thus, the contrast obtained is the result of changes in the fluorescence intensities due to variation in the $\mathrm{Ca}^{2+}$ levels. Another critical factor is the $\mathrm{pH}$, which affects the amount of anionic chromophore concentration. Ideally, it is advised to optimize and maintain these parameters during in vivo imaging. Experimental setup for imaging brain activity in the cortical layer $2 / 3$ neurons in awake transgenic mice consisted of alternating excitation using $3 \mathrm{PF}$ microscopy within a microsecond time scale. Investigating the dependence of layer in response to oriented gratings moving in specific directions (arrow marks above each trace) and their orientation tuning curves in polar plots. Randomization of each stimulus direction in each trial caused discontinuities in $(\Delta F / F)$ traces. $(\Delta F / F)$ scale bars correspond to $100 \%$ and the time scale bar corresponds to $10 \mathrm{~s}$ as shown in the bottom right panel (Yildirim et al. 2019)

3PF signal intensity on excitation wavelength led to the determination of optimal excitation conditions that provide a high signal-to-noise ratio (SNR) with low excitation power. The study visualized the brain activity with $2 \mathrm{PF}$ and $3 \mathrm{PF}$ by varying one wavelength while keeping the other constant. The results suggested that the $3 \mathrm{PF}$ signal increased with an increase in the excitation wavelength (Ouzounov et al. 2019).

The application of 3PF microscopy for in vivo imaging of astrocytes in deep mouse brain was investigated using sulforhodamine 101 (SR101) labeled blood vessels. The optimal excitation wavelength was selected based on the wavelength-dependent $3 \mathrm{P}$ action cross-section $\left(\sigma_{3}\right)$ measurement of SR101. While the signal-to-background ratio (SBR) in $2 \mathrm{PF}$ is limited to just $910 \mu \mathrm{m}$, an imaging depth of $1340 \mu \mathrm{m}$ was achieved using $3 \mathrm{PF}$ which may be limited due to signal depletion and SBR limit. Based on the proportionality dependence of wavelength-dependent $\sigma_{3}$ and imaging depth Liu et al. (2019) demonstrated that for SR101, $\sigma_{3}$ reached its maximum on excitation with $1620 \mathrm{~nm}$. Second, a diode-pumped $\mathrm{Nd}$ :YLF laser generating extremely short pulses at $1047 \mathrm{~nm}$ 
was used for 3P excitation of several commonly used biological fluorophores (Wokosin et al. 1996). Third, the GradientIndex lens-based 3P endoscope provided a compact method of diagnosing tissues with a lower cost and higher clinical accuracy (Huland et al. 2013). Fourth, a 3P widefield microscopy technique that can excite chromophores such as quantum dots and channel-rhodopsin and achieve a penetration depth up to $700 \mu \mathrm{m}$ can overcome the limitations of conventional 3PF microscopy (Rowlands et al. 2017). Quantum dot imaging using $3 \mathrm{P}$ widefield microscopy in mouse brain (quantum dots were injected via tail veins and blood vessels) improved imaging depth and resolution when combined with adaptive optics techniques (Sinefeld et al. 2015).

\section{Second-harmonic generation (SHG) microscopy}

SHG microscopy is a label-free technique used in cellular and tissue imaging as it can spatially resolve sub-cellular details in 3D with high molecular contrast (Campagnola 2011; Mazumder et al. 2014; Zipfel et al. 2003). The presence of anisotropic, hyper-polarizable, non-centrosymmetric molecules within the specimen makes it easier to perform SHG imaging (Mazumder et al. 2014; Mazumder et al. 2012). Collagen is one of the commonly studied molecules used in SHG imaging (Tilbury and Campagnola 2015). Among the twenty different forms of collagen, type-I is a chief constituent of connective tissues and extracellular matrix (Zipfel et al. 2003). SHG microscopy allows in vivo, non-labeled, highresolution deep tissue imaging with minimal photodamage and photobleaching. Analysis of the SHG signals helps in the study of the distribution and structural orientation of the SHG active molecules within the specimen. On enhancing the resonant SH signals of different electronic levels, signals can be extracted from specific molecules which enable the construction of a map of the distribution of these molecules across a biological sample.

SHG microscopy allows high-resolution imaging of astrocyte processes and the surrounding parallelly arranged myelin sheaths that are wrapped around the axons. This method can be used to diagnose a neurodegenerative disorder that targets the myelin sheath causing demyelination and could be used in the early detection of demyelination in various disease conditions (Huff et al. 2008). In another study, tumor (glioblastoma) and normal brain tissue sections were examined in vivo using the combination of $2 \mathrm{PF}$ and SHG techniques, which were then compared to the standard H\&E staining method. SHG signals generated from the branched blood vessels associated with neurons highlighted the cortex region of the brain. Glioblastoma was characterized by the presence of glomerular vessels and high cellularity. It was observed that during metastasis, strong SHG signals were obtained due to the formation of a collagen-rich matrix around the tumor, thus establishing SHG microscopy as a reliable alternative to the standard
H\&E staining technique (Poulon et al. 2018). Also, Ischemia is a condition that can disrupt the cytoskeleton of neurons. In a study, changes occurring during the early stages of ischemic development were studied by brain tissue imaging using polarization-resolved SHG (PR-SHG) microscopy. Neuroglial cells were subjected to a state of oxygen and glucose deficiency to induce ischemia. The SH signal was maximum when the incident linearly polarized light was parallel to the axon and minimum when it was perpendicular to the neuron. PR-SHG imaging was performed at regular intervals of time and an anisotropy histogram was plotted from the ischemia-induced neurons. Changes in the height and width of the histogram peak about $120 \mathrm{~min}$ after the experimental procedures suggested the onset of the disease (Psilodimitrakopoulos et al. 2013).

Parkinson's disease (PD) generally affects the dopamineproducing cells in the central nervous system (CNS) ultimately affecting the movement of an individual. SHG microscopy was used to identify the conformation of $\alpha$-synuclein, a protein that is known to form aggregates within the neuron in PD conditions (Psilodimitrakopoulos et al. 2013). Since $\alpha$ synuclein cannot produce SHG signals, this protein was conjugated with an SH active probe or dye. SHG microscopy is highly sensitive to the structural orientation of molecules; therefore, the intensity of the SHG signal obtained depends on the tilt angle of the dye to the surface of the protein. This method was used to study the role of conformation of $\alpha$ synuclein in the formation of aggregates and their role in PD. In a neuronal cell model, BIOD303 binds to a specific conformation of $\alpha$-synuclein and reduces its aggregation. This technique can also be used to classify ligands based on the confirmation of the protein they bind to or to perform further studies on the therapeutic uses of this effect (Moree et al. 2015). Using $2 \mathrm{PF}$ and SHG microscopy, ex vivo brain slices of a transgenic wild-type mouse with $\mathrm{AD}$ were studied. Four major structures were visualized including, senile plaques, microtubules, blood vessels, and lipofuscins. The senile plaques were seen only in the specimens that were affected by $\mathrm{AD}$ which emitted a strong fluorescence and a weak SHG signal. SHG emissions of microtubules located near the plaques were used to determine the length and density of the microtubules and it was observed that the mice with short microtubules manifested AD. Abnormal features such as axonal varicosity, loss of spines in dendrites, shaft atrophy, and sprouting have been reported in the disease condition. However, SHG microscopy revealed that apical dendrites located near the disease lesions showed no abnormality (Kwan et al. 2009). The structural characterization of insulin amyloid fiber formed in conditions like $\mathrm{AD}$ and $\mathrm{PD}$ was performed. These non-centrosymmetric crystalline fibrils are different from their amorphous core proteins and can be analyzed by SHG microscopy. On SHG imaging of the amyloid fibrils, the core of the insulin spherulites exhibited a weak response, thus, 
confirming its amorphous nature. This method can be used for in vivo imaging and characterization of amyloid fibers to diagnose diseases (Johansson and Koelsch 2017). Native brain tissues display strong SH signals in certain regions. In a study, hippocampal tissue sections and ex vivo CA3 pyramidal neuronal cell culture were imaged to identify the origin of these SHG signals (Dombeck et al. 2003). It was concluded that the SHG dipole radiation is produced only when the microtubules were aligned parallel to the excitation polarization and no signal is obtained when it is aligned perpendicular to the excitation polarization. This is presumed to be due to hyperpolarization. SHG images of the native hippocampal tissue sections illuminated a large curved bundle of mossy fiber axons (a dense unmyelinated axon bundle) (Fig. 4E, F). Similar signals were obtained from the dentate gyrus granular neuron that innervates the $\mathrm{CA} 3$ pyramidal neurons. On magnification and closer examination of the SHG images, the SHG signals were found to be strong in the axonal regions and non-existent in the dendritic and somatic regions. Tau immunostaining technique (which stains only the axon) resulted in an image identical to the SHG image obtained, further verifying the fact that SHG signals arise only from the axons (Fig. 4G). To find the actual origin of the SHG signals, the tissues were treated with nocodazole (depolymerizes microtubules) and cytochalasin D (depolymerizes actin filaments). SHG signals of the nocodazole treated specimen decreased by $39 \%$ of its original value while that of the cytochalasin $\mathrm{D}$ treated specimen remained unchanged. The study concluded that SHG signals
CONTROL
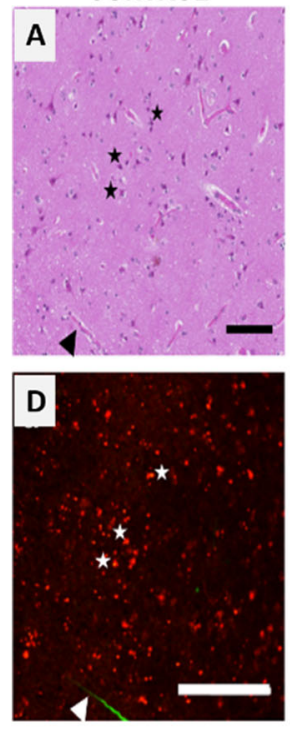

METASTASIS
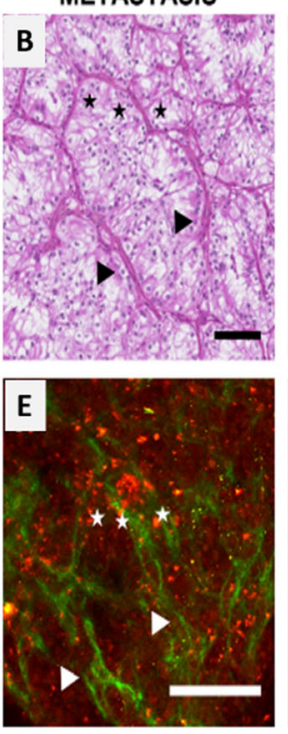

GBM
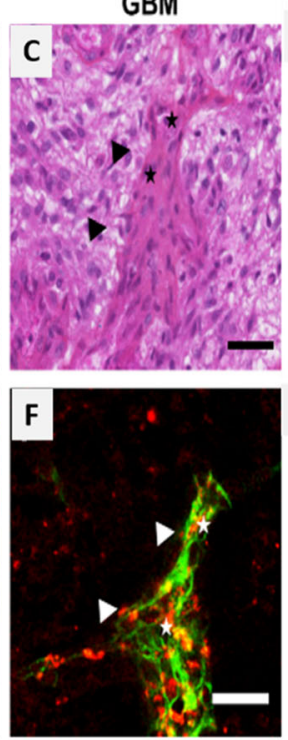

Fig. 4 (A-C) Images showing optical sections obtained after H\&E staining (a gold standard in neuropathology) and (D-F) merged 2PF (red) and SHG (green) images, in control (normal brain tissue), metastasis, and GBM (glioblastoma). Stars: neurons; arrow: brain vessels (Poulon et al. 2018). (G) SHG image of a neuron stained with FM4-64. (H) Averaged line scans represented with black traces (left-axis scale) are from the hippocampus of native brain tissues and neuronal cells fundamentally originate due to the uniform polarity of the microtubules in the axons (Dombeck et al. 2003; Sacconi et al. 2006). Thus, due to its specificity and high sensitivity, SHG microscopy can be used as a novel method for imaging in the field of neuroscience and also has great potential to contribute to its future growth and development.

\section{Third-harmonic generation (THG) microscopy}

THG microscopy is used to reveal cellular morphology and molecular information of biological samples (Weigelin et al. 2016; Zhang et al. 2017; Gualda et al. 2008). In the brain tissues, the contrast mainly arises from the interfaces between lipid-rich molecules (Gualda et al. 2008). Since the signals are generated from the sample, use of exogenous contrasting agents can be avoided, which is one of the advantages of THG microscopy. As a single laser source is required for THG, it is compatible with $2 \mathrm{PF}$ and SHG imaging. This technique can achieve high penetration depth, label-free imaging, low phototoxicity, diffraction-limited resolution, and intrinsic 3D sectioning. Brain tissue morphology is studied by THG imaging using a non-invasive probe (Witte et al. 2011). Since the lipid-rich structures such as the axons and dendrites constitute a large portion of the brain, in vivo deep tissue imaging of live neurons can be performed. Signals arising from these structures can be used to reconstruct high-resolution images of the neurons without the usage of fluorescent dyes or genetic
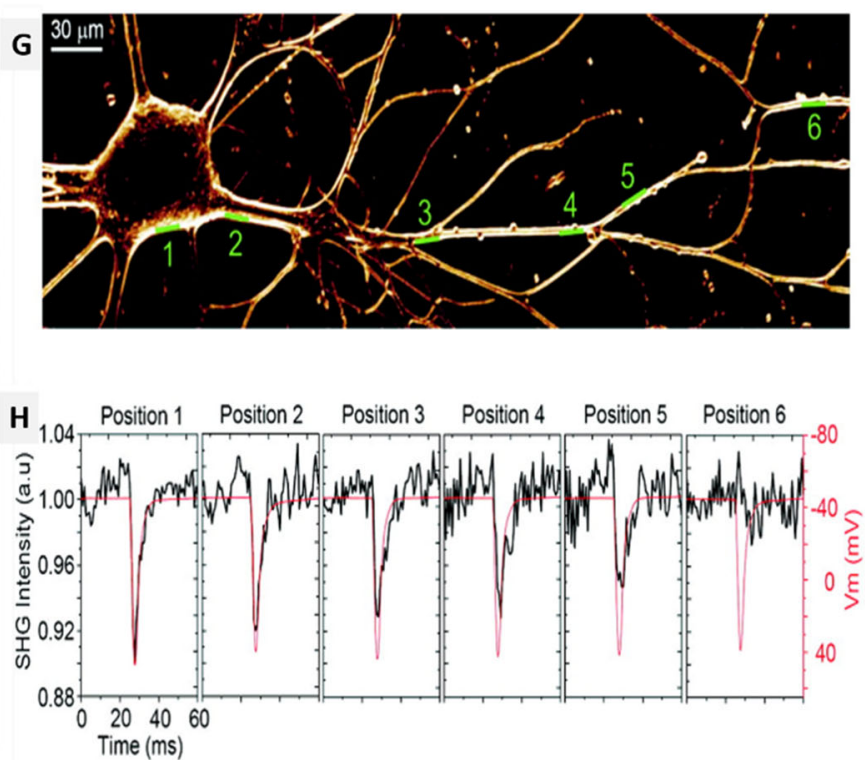

normalized intensity plots of SHG emission versus time at the membrane positions shown in (G). The red traces (right-axis scale) are the Vm from the recording electrode at the soma (Sacconi et al. 2006). Scale bars: $100 \mu \mathrm{m}$ in (A, B, D, E) and $40 \mu \mathrm{m}$ in (C, F) (Poulon et al. 2018; Sacconi et al. 2006) 
probes. Abnormal cholesterol accumulations in the brain are found in senile plaques and can be a characteristic of $\mathrm{AD}$. THG imaging of lipids at cellular resolution with intrinsic contrast is useful for the investigation of several disorders (Gualda et al. 2008).

Neuronal dysfunction, one of the factors leading to neurodegeneration can be studied by visualizing the neurons via THG microscopy. Caenorhabditis elegans (C. elegans) was used as a study model to identify the THG specificity for neuronal imaging (Gualda et al. 2008). C. elegans were subjected to programmable cell death by inducing gain-offunction mutations in genes coding for the degeneration family. The THG signals arising from control and degenerative neuronal regions showed a clear distinction between the two; where the degenerated neurons produced higher intensity signals. In vivo THG imaging revealed the biological processes that occur in neuronal degeneration and facilitated the detection of specific structural features within them (Gualda et al. 2008). Multiple sclerosis (MS), a neurological condition often caused due to loss of myelin function as the result of damage and inflammation, can be studied based on changes in the neuronal structure. In vivo and ex vivo THG imaging of myelin in the CNS of mice and zebrafish models suggested the myelin specificity of THG signals. While THG signals are predominantly formed due to the lipid-rich structures in the brain, this study established the generation of signals from myelin as well. The geometrical properties of myelin such as scattering and thickness generated myelin-specific THG signals. For in vivo imaging, the source of contrast was enhanced using a laser source of low repetition rate and high peak power (Zhang et al. 2017). THG microscopy can be a potential highthroughput drug testing candidate along with other techniques for animal models and is an emerging tool for visualizing myelinated neurons in both CNS and PNS (Farrar et al. 2011). Morphological changes in myelin induced during the process of postnatal development and degradation are other potential areas for the use of THG microscopy (Lim et al. 2014).

Lim et al. (2014) monitored the morphological changes in myelin through the process of postnatal development and degradation. Live cell THG imaging was performed using cultures of the myelinated and non-myelinated axon of rat dorsal root ganglion (DRG) and Schwann cells. Schwann cells were visualized as thin elongated sections with lengths between 60 $-160 \mu \mathrm{m}$. It was observed that the signals generated from the myelinated cells were higher than those generated from nonmyelinated DRG neurites. To further verify that the obtained THG signals were derived from myelinated fibers, DRG neurons with and without Schwann cells were cultured and imaged. It was noticed that the elongated segments emitted a strong THG signal only in the myelinated samples. Based on the obtained THG signals, the locations of hemi-nodes and nodes at the adjacent internodal junctions can be pinpointed.
An intact in vivo analysis of nerves using epi-detection of THG signals was performed to examine the feasibility of THG microscopy. The dynamics of myelination in Schwann cells, both in vivo and ex vivo (Fig. 5) were explored. Images of myelinated fibers allowed the visualization of the collagenous endoneurium and perineurium. THG signal strength is sufficiently strong from myelinated axons in intact excised tissues as well as in live animal models. Thus, signals generated specifically from the myelin sheath enable the visualization of diverse myelin domains in biological systems.

Cortical demyelination plays a crucial role in the development of neurodegenerative conditions such as MS. In a study, THG signals from the neurons of transgenic murine models were evaluated, and the imaging of cortical myelin was carried out both in vivo and ex vivo. THG signals are generated due to the mismatches in the refractive index between the cytoplasm and lipid membrane of the neurons in the CNS and PNS. The image contrast was also employed to study the myelinated axon of gray matter in the mouse brain. In transgenic mice, Thy1-yellow fluorescence protein, YFP (Thy1-YFP), and CNP-GFP (2',3'-cyclic nucleotide 3'-phosphodiesterase-green fluorescent protein) were used, which expressed YFP in the cytoplasm under the promoter Thy 1 and membrane attached GFP in myelinated cells via the CNP promoter respectively (Redlich and Lim 2019). Further analysis suggested that the THG signals originate from myelinated axons (Fig. 6A). The experiments also involved studying the depth-dependent orientation of myelin fibers in a fixed transverse section of the brain. Visualization of tangential and radial fibers was achieved without directional bias (Fig. 6B) and with substantial depth-dependent variations to the total and relative abundance of these fibers. Additionally, quantitative analysis was performed by selecting $3 \mathrm{D}$ volume at three different depths in the cerebral cortex (I, II, III in Fig. 6B respectively) and semiautonomous tracing of the myelinated axon. The axial projection of the traced THG-positive axon revealed that the density of radical fibers increased consistently with depth, and some fibers aggregated to form bundles in the deeper layers of the cortex (Fig. 6C arrowhead). Hence, in vivo imaging and 3D reconstruction of the myelin network in the mouse brain using THG signals can aid in neurological research. One of the advantages of THG imaging is that it can be performed on existing multiphoton microscopes with the simple addition of a suitable, albeit nonstandard, femtosecond laser source and the appropriate detection filters (Farrar et al. 2011). Application of THG microscopy can be extended to the study of remodeling in myelin from fresh tissues using biometric measurement, revealing axonal and abaxonal membranes differentiation from the large-caliber axon, and can be applied for morphometric analysis of myelin sheath. Hence, THG microscopy can be used to describe micro-architecture and identify neuronal degradation (Redlich and Lim 2019). 
A

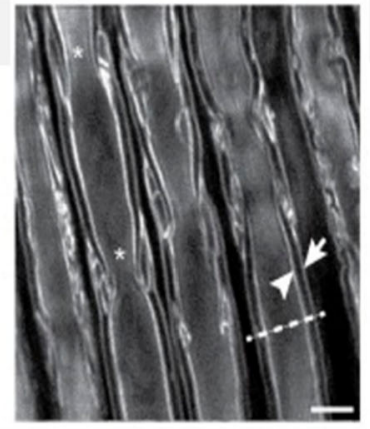

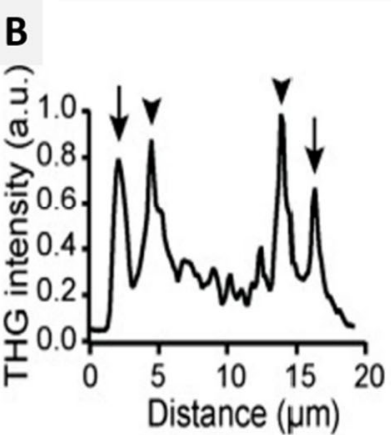
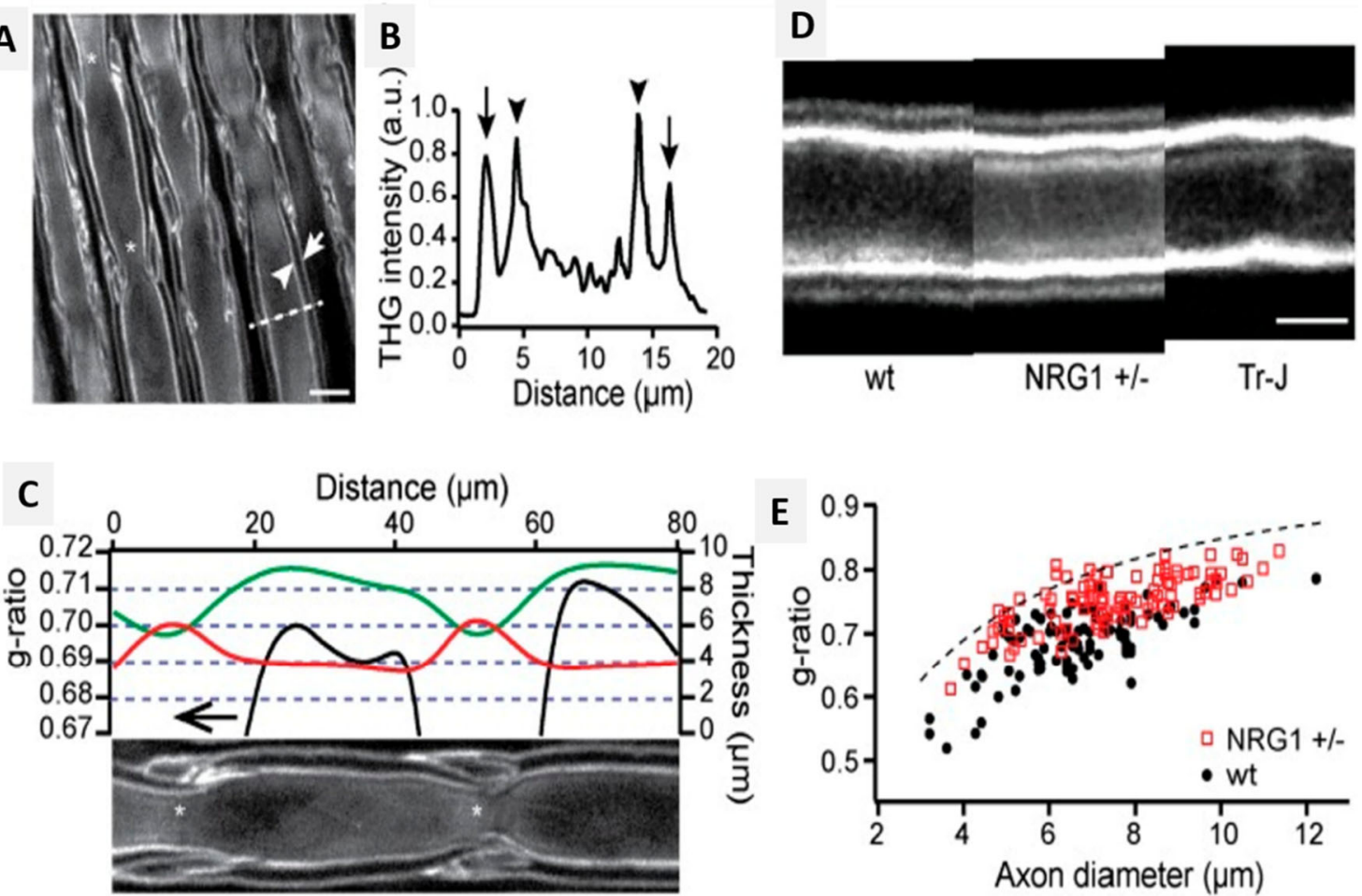

Fig. 5 Ex vivo imaging of myelin sheath with THG. (A) Image shows the lateral section of non-teased rat sciatic nerve, adaxonal (arrowhead) and abaxonal (arrow) membranes (scale bars, $10 \mu \mathrm{m}$ ). (B) Image shows the THG intensity profile for the dashed line in (A). (C) The thickness of axon (green) and myelin sheath (red), and the g-ratio (black) along the internode shown at the bottom. (D) Unfixed, teased sciatic nerves of WT,

\section{Coherent anti-Stokes Raman scattering (CARS) microscopy}

High-contrast imaging can be obtained using SHG and THG microscopy; however, obtaining a chemical contrast using these techniques is challenging (Evans et al. 2005). This limitation can be resolved using the CARS technique (Zumbusch et al. 1999). CARS is a label-free, third-order, nonlinear, fourwave mixing process that uses the Raman vibrational frequency of a specific chemical bond within the specimen to create chemical contrast. Dysregulation of lipid metabolism is one of the factors responsible for a neurological disorder. Damage and degradation of myelin sheath can lead to reduction or obstruction in nerve impulse conduction resulting in neurological conditions (Le et al. 2010). CARS microscopy is used to identify and image lipids and proteins by exciting the $\mathrm{CH}$ vibrations at 2600-3000 $\mathrm{cm}^{-1}$ (Mazumder et al. 2013). Kiskis et al. (2015) studied the human AD-affected brain tissue sections to determine the spatial distribution of lipids in the prefrontal cortex using CARS microscopy. The lipids in the cells and tissues were visualized by excitation and probing $\mathrm{CH}$ stretching vibration of fatty acid. CARS signal at $2840 \mathrm{~cm}^{-1}$ corresponding to long-chained triglycerides was used for the spectral analysis of the $\mathrm{CH}$ vibrational region. Constructing
NRG1 type III ${ }^{+/}$, and Tr-J mice with similar caliber. Abaxonal membrane position shifts due to the myelin thickness difference (scale bar, 5 $\mu \mathrm{m})$. (E) The scatter plot of g-ratio as a function of axon caliber in WT and NRG1 type $\mathrm{III}^{+/}$animals. The dashed line corresponds to the g-ratio with $0.9-\mu \mathrm{m}$ gap between the adaxonal and abaxonal membranes (Lim et al. 2014)

pixel-wise ratios of the CARS signals at the symmetric and asymmetric $\mathrm{CH}$ vibrations provided the lipid fluidity (degree of unsaturation) throughout the plaque and its circumambient regions. The CARS images revealed the accumulation of lipids, primarily in areas of fibrillar $\mathrm{A} \beta$ plaques as shown in Fig. 7. Lipid fluidity could help establish the extent of release of toxic $A \beta$ oligomers from the fibrillar plaques. Since CARS microscopy does not involve immunostaining, the $\mathrm{CH}$ ratio is used as indirect toxicity maps (Kiskis et al. 2015). The regions identified as large coalescent lipid aggregates comprise lipids with higher fluidity, while the protein-lipid interaction sites of lamellar regions consist of ordered lipids.

Myelin is largely composed of lipids (70\%) and proteins (30\%) (Mazumder et al. 2012), which facilitates imaging using CARS microscopy. Studies have demonstrated CARS imaging of myelin fibers in mouse brains under in vivo and ex vivo conditions (Kiskis et al. 2015; Fu et al. 2008; Brideau et al. 2019). The presence of a high density of ordered $\mathrm{CH}_{2}$ groups in myelin sheath facilitates the generation of a large CARS signal. Imaging myelin without the necessity of labeling eliminates the labor-intensive sample preparation processes. White matter in fixed slices, fresh tissues, and live animals can be imaged via CARS microscopy by detecting symmetrical $\mathrm{CH}_{2}$ vibrations (Fu et al. 2008; Imitola 
A
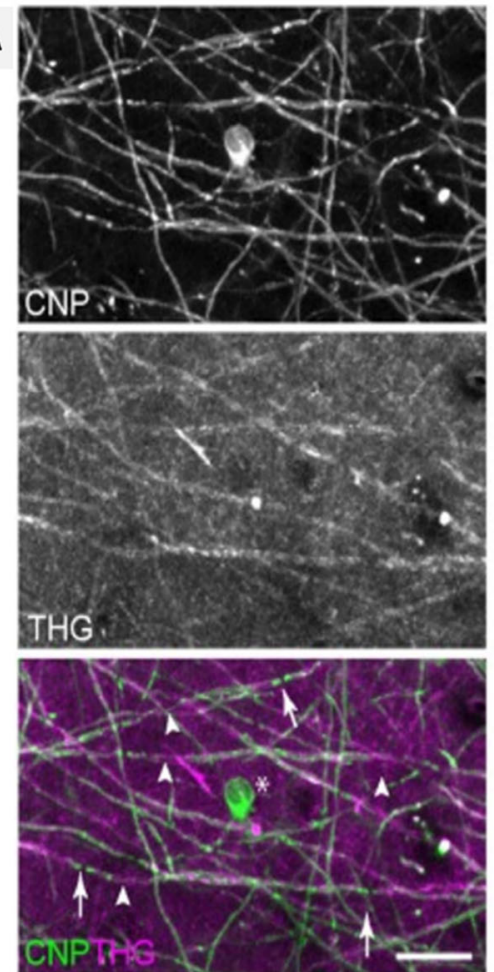

Fig. 6 THG imaging in mouse brain (A) co-registration of CNP-GFP and THG signals $(B, C)$ tangential and radial fiber distribution based on depth-dependence (B) volumetric rendering of THG stacks in the range of $0-800 \mu \mathrm{m}$ of the cortex. I, II, and III indicate $0-100 \mu \mathrm{m}, 200-300 \mu \mathrm{m}$,

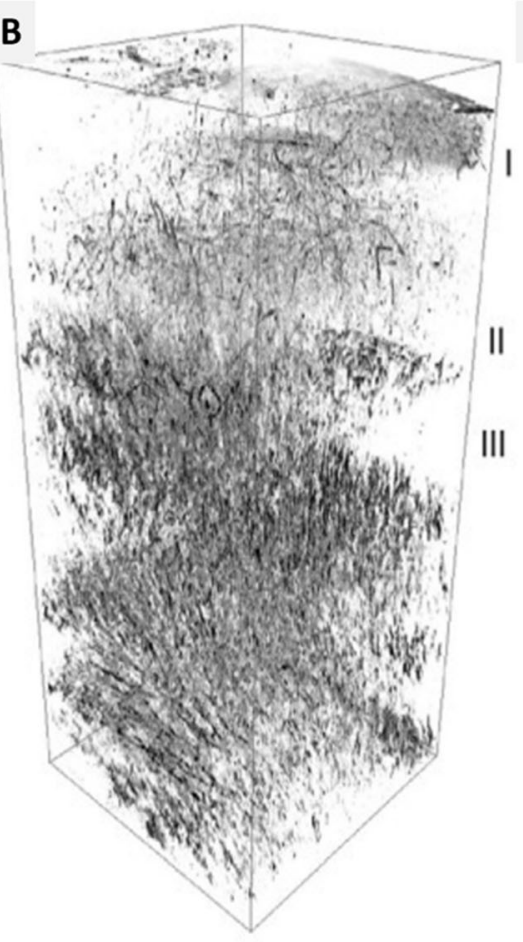

and 300-400 $\mu \mathrm{m}$ below the dura, respectively. (C) Transverse and axial projections $\mathrm{XY}$ and $\mathrm{XZ}$ respectively of traced axon showing lattice structure and fiber bundles; scale bar $=20 \mu \mathrm{m}$ (Redlich and Lim 2019)
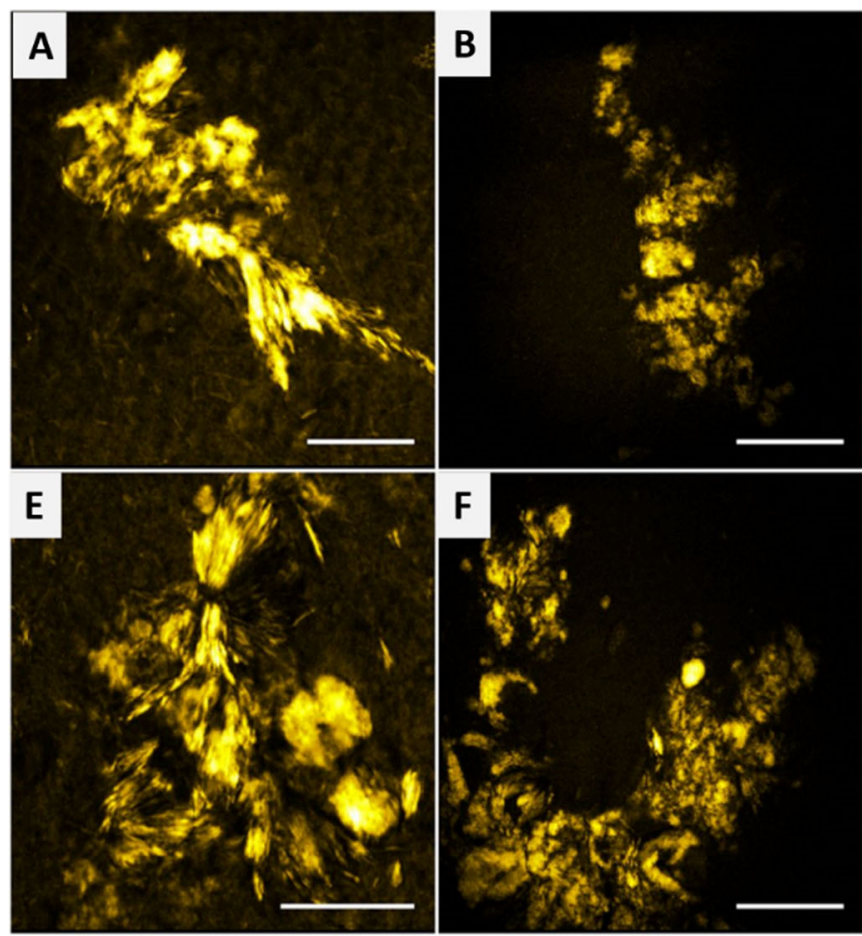

Fig. 7 Image showing the heterogeneity in lipid aggregate composition from AD brain tissue samples from 2 patients imaged with CARS at 2840 $\mathrm{cm}^{-1}$. Lamellar structures (seen in A, E, and G) and coalescent structures of different constructs most likely arise from lipid micelles initially c<smiles></smiles>
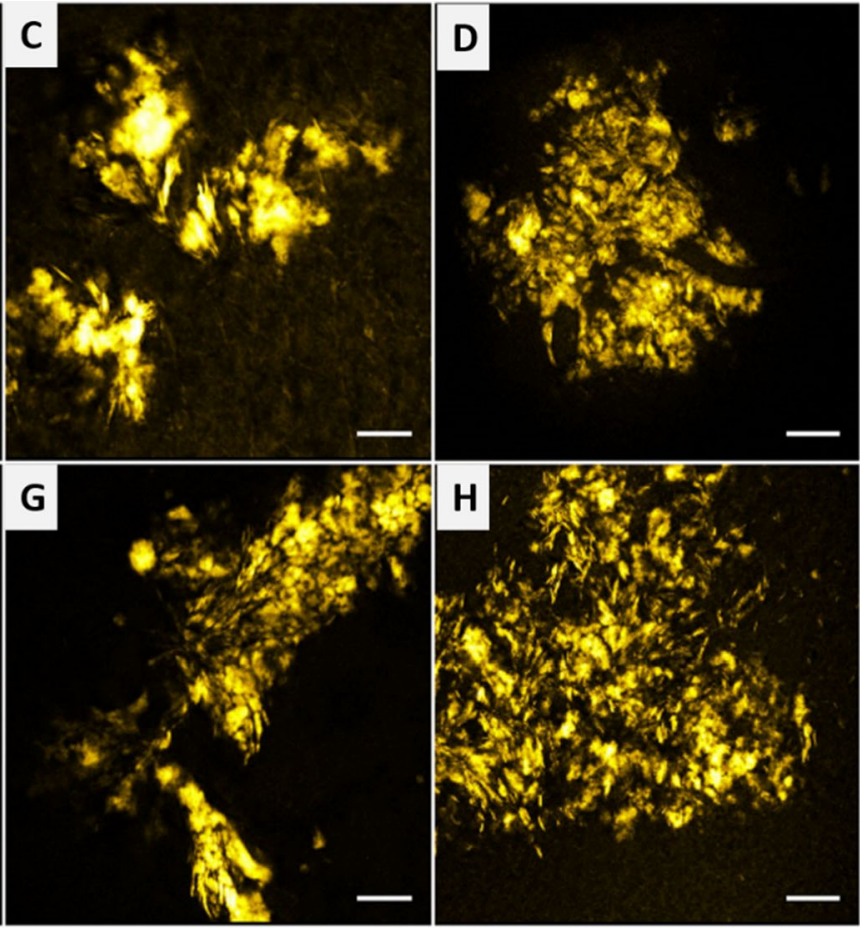

decorating the $A \beta$ fibrils or $A p o E$ particles, alternatively from lipid microvesicles shed by microglia. (A-D) Patient $1,(\mathrm{E}-\mathrm{H})$ patient 2 . Scale bars, $25 \mu \mathrm{m}$ (Kiskis et al. 2015) 
et al. 2011). Epi-detected CARS microscopy technique, when used to image sub-cortex white matter of the mouse brain proved to be a feasible technique for in vivo studies (Kiskis et al. 2015). Fu et al. (2008) performed in vivo imaging of the mouse brain (Fig. 8A, B) using multimodal CARS microscope and simultaneous tissue imaging by CARS and 2PF (Fig. 8C, D) to identify the organization between cells and axonal tracts. In vivo imaging of the parietal cortex was limited to a depth of $30 \mu \mathrm{m}$ due to the low optical penetration depth. However, upon aspiration of gray matter, the white matter was imaged by CARS microscopy showed bundles of myelinated axon in one direction without the use of labeling as shown in Fig. 8B. Imitola et al. 2011 illustrated a new, multimodal CARS imaging technique for investigation of myelin structure using reflectance and fluorescence confocal imaging where the CARS signal is tuned to the $\mathrm{CH}_{2}$ vibration of lipids. It was performed on animal models with chronic experimental autoimmune encephalomyelitis (EAE), a condition leading to the demethylation of neurons in the brain and spinal cord. In this study, CARS microscopy was used in combination with other non-invasive techniques such as reflectance to reveal the changes in the lipid signals in myelinated and unmyelinated regions of the EAE mouse brain using thick living tissue sections (Brideau et al. 2019). CARS microscopy has emerged as a promising, label-free imaging modality facilitating 3D sectioning real-time, chemically selective imaging both in vivo and ex vivo (Lee et al. 2015). CARS microscopy has shown potential clinical applications and may replace traditional histopathological techniques such as marginally invasive micro-endoscopy for the diagnosis of neuropathologic neoplastic and non-neoplastic brain diseases (Fu et al. 2008). However, CARS microscopy suffers from a non-resonant background and mostly reveals information on $\mathrm{CH}$-stretching. Importantly, the CARS microscopy technique is not possible
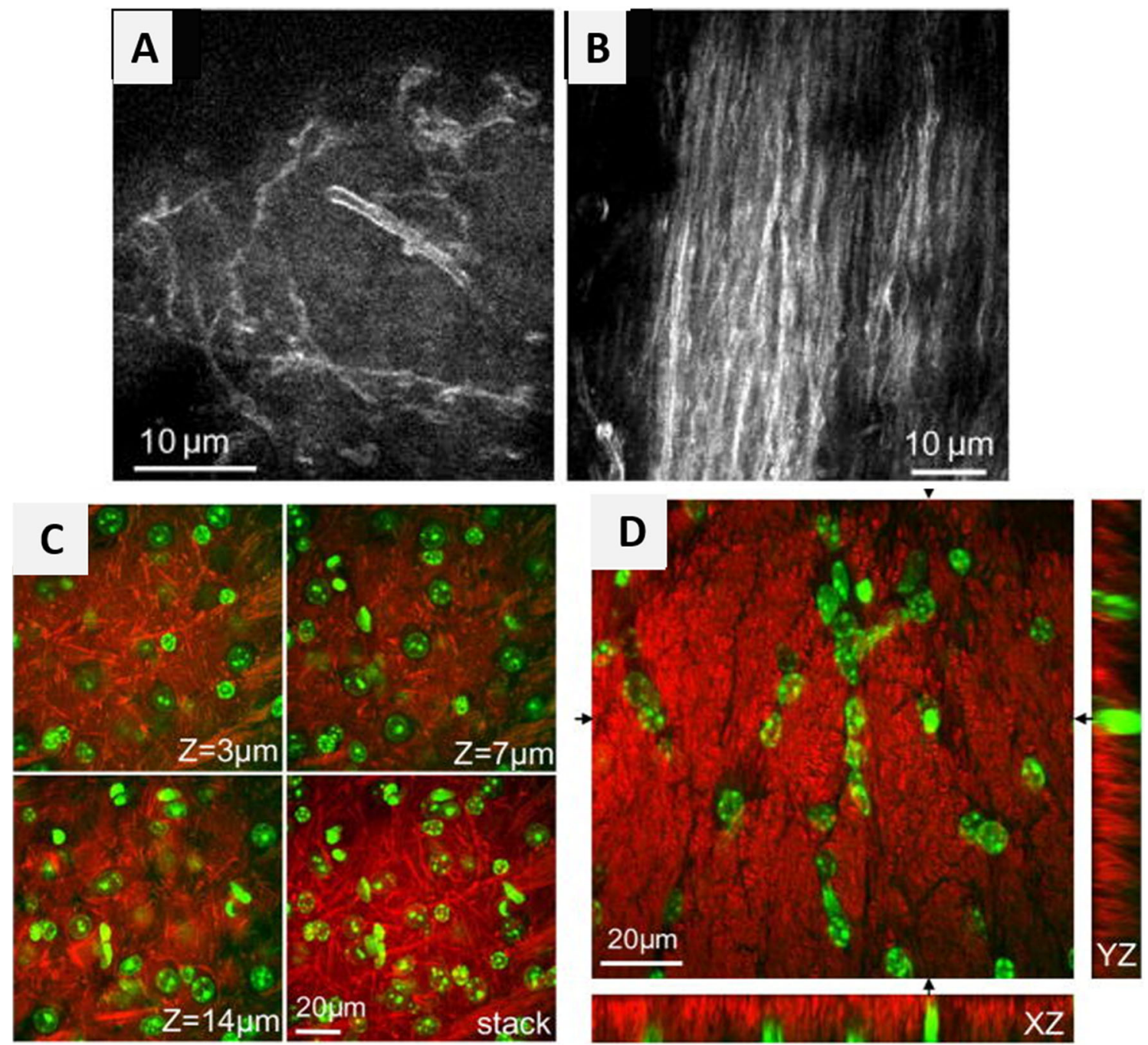

Fig. 8 (A and B) In vivo imaging of the mouse brain. CARS image of (A) parietal cortex (B) bundles of myelinated fibers in the sub-cortex white matter; (C and D) ex vivo imaging with slices of white and gray matter by

simultaneous CARS imaging of myelin sheath and 2PF imaging of nuclei. Red: signals from CARS; green: signals from $2 \mathrm{PF}$ of Hoechstlabeled cell nuclei (Fu et al. 2008) 


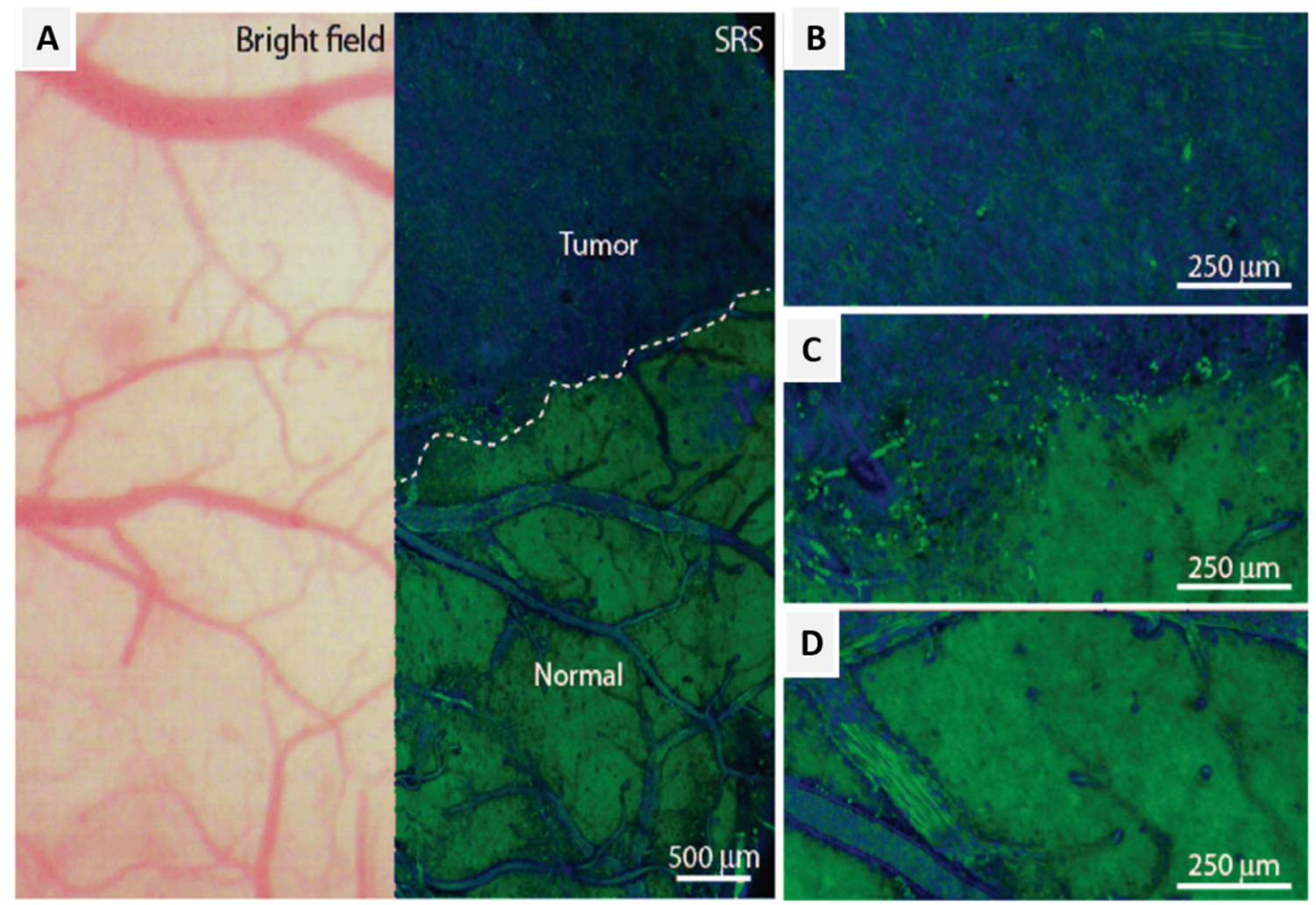

Fig. 9 In vivo SRS microscopy images of human GBM xenografts. (A) Bright-field microscopy imaging appears to be nearly normal. In contrast, SRS microscopy within the same region of focus shows distinctions between tumor-infiltrated and non-infiltrated areas of the brain (normal), clearly depicting the brain/tumor interface (dashed line). (B-D) showing magnified views (B) within the tumor, $(\mathrm{C})$ at the brain/tumor interface, and (D) within the normal brain (Ji et al. 2013) to use in the fingerprint region, which is very essential for much biochemical information.

\section{Stimulated Raman scattering (SRS) microscopy}

The stimulated emission (SE) phenomenon is one of the basic working principles of laser which was discovered in 1917 by Einstein. SE was first used by Hell and Wichmann (1994) to achieve beyond diffraction-limited resolution in light microscopes. In recent years, several SE based optical imaging modalities have emerged for the detection of the so-called undetectable chromophores (Min et al. 2009) and fluorescently labeled samples (Das et al. 2019a; Das et al. 2019b). However, fluorescence labeling can be difficult in some small intracellular molecules such as signaling peptides, neurotransmitters, and metabolites as the size of the fluorescent molecule is much larger than that of the molecule of interest. Also, fluorescent dyes often undergo photobleaching over time. In contrast, a label-free chemical imaging technique based on Raman scattering offers molecular specificity on the biochemical and biophysical composition of cells and tissues (Klein et al. 2012; Ogawa et al. 2009). Notably, due to the shorter excitation wavelength, spontaneous Raman scattering signals of interest overlaps with unwanted Raman signals and background auto-fluorescence (Hamada et al. 2008). An alternative vibrational imaging technique based on coherent Raman scattering (SRS) offers a higher cross-section of several orders of magnitude, as compared to spontaneous Raman scattering. For the past few years, SRS microscopy has become an indispensable tool for molecular fingerprinting and contrast in the field of neuronal imaging (Shin et al. 2019; Ji et al. 2013). SRS imaging at a Raman signal of $1660 \mathrm{~cm}^{-1}$ represents the $\mathrm{C}=\mathrm{C}$ bonds of unsaturated lipids, and the amide I band of proteins showed that the spectral shift of amide I band of $\beta$ sheets make it possible to distinguish $A \beta$ from normal proteins and lipids. Hence, this provides a label-free method to study AD and other diseases related to protein misfolding. In the animal and human nervous system, acetylcholine plays an important role as a neurotransmitter to activate muscles. SRS imaging of neurotransmitter acetylcholine (ACh) was performed in frog muscle with a frequency-modulation, spectral-focusing SRS technique (Fu et al. 2017). To test the SRS imaging technique, $\alpha$-BTXAlexa488 was used to stain ACh receptors in the neuromuscular junctions. The ACh-receptors were concentrated in synaptic vesicles and appear wider in the fluorescence image. A study investigated the rapid imaging of $A \beta$ plaques in brain tissues of 
A Brain slice

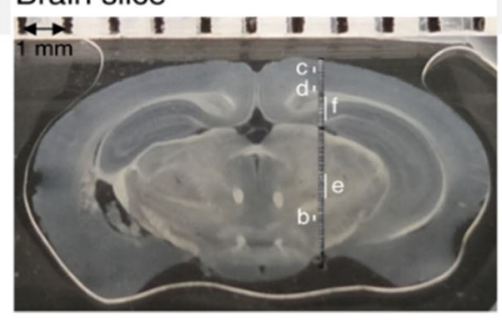

B

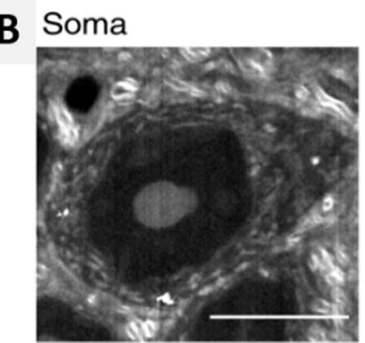

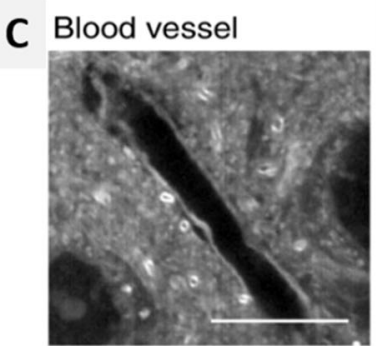

D Axon

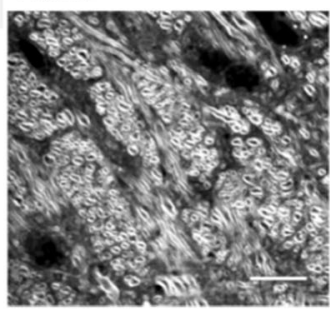

E

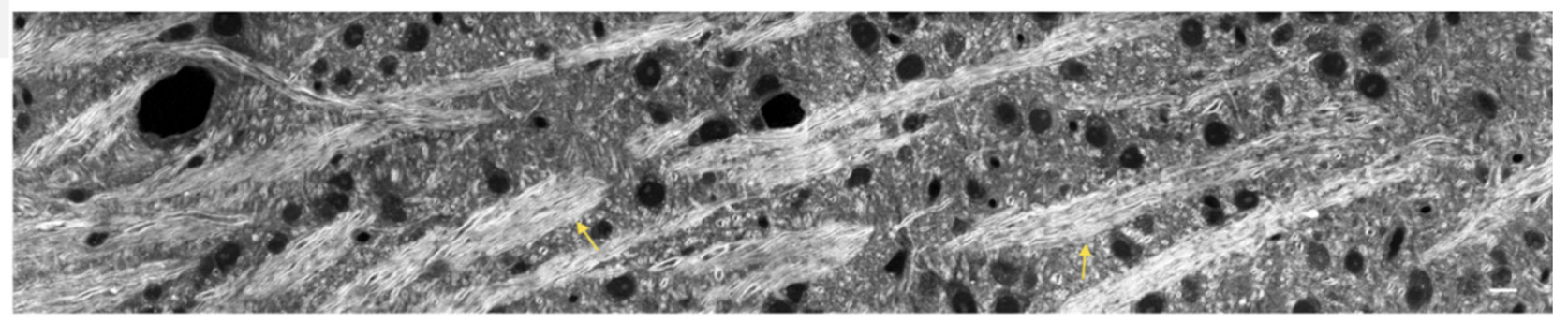

F Alveus of the hippocampus

Subiculum

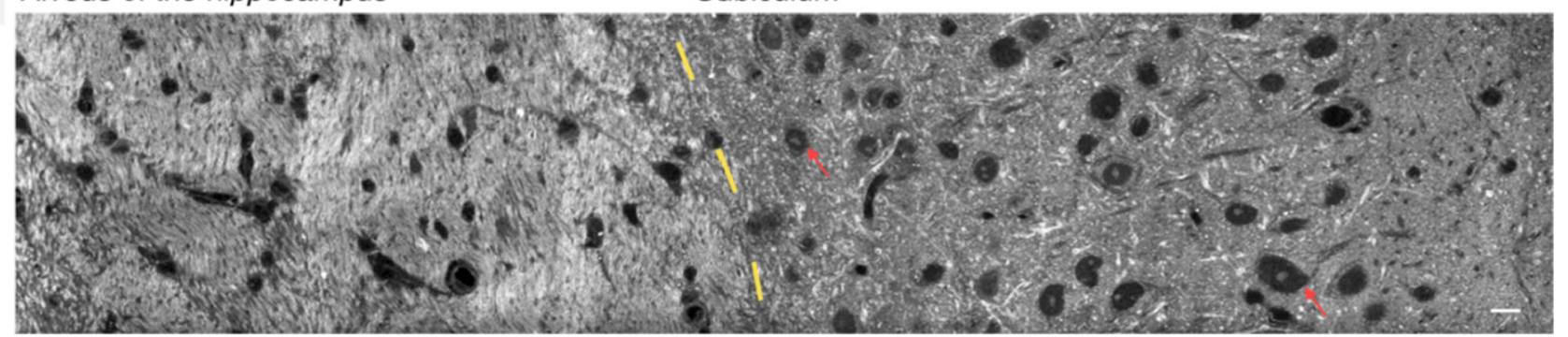

Fig. 10 Visible SRS imaging of an unprocessed brain tissue section from a C57 wild-type mouse. (A) Overview of a coronal section of the brain slice. SRS inspected area is shown. (B-D) Enlarged views that illustrate the architectures of the soma (B), blood vessel in the cortex (C), and fiber bundles in white matter (D), with their locations marked by white lines (B-D) in (A), respectively. (E, F) High-resolution SRS imaging of brain areas corresponding to $(\mathrm{E}, \mathrm{F})$ indicated in $(\mathrm{A})$, respectively. Scale bar is $10 \mu \mathrm{m}$ (Bi et al. 2018) transgenic mouse models with AD using CARS and SRS microscopy (Fu et al. 2017). The major emphasis was on SRS microscopy due to its ease in conducting qualitative analysis and maintaining Raman spectra undistorted. Again, Ji et al. (2018) demonstrated the feasibility of SRS microscopy in distinguishing normal and tumor conditions by utilizing the Raman signal originating from macromolecules for imaging the mice cortex. The cortical imaging of tumor engrafted transgenic mice was performed using a coverslip to create a cranial window to achieve non-invasive imaging. Bright-field and SRS imaging of brain tumors provided substantial evidence to prove the effectiveness of the SRS technique. Tissues within the tumor regions appeared indistinguishable from the normal samples when imaged using bright-field (Fig. 9A), while SRS images showed distinctive features that can be used to differentiate between normal and tumor samples (Fig. 9B) (Ji et al. 2013). Further ex vivo imaging supported their hypothesis that the individual cells at the brain/tumor interface are neoplastic given the paucity of the cells at their imaging depth. SRS microscopy is proven to be an efficient method for identifying tumors, thus paving the way towards a better understanding of the disease.
Visual access to the mouse brain is essential for in vivo studies to understand the structural and functional features. Conventionally, satisfactory depth for imaging the mouse brain was achieved by opening the opaque skull which may alter the physiological conditions in the brain. Label-free approaches such as SRS microscopy have presented a promising future for higher-resolution brain imaging, through advanced skull and tissue clearing methods (Chen et al. 2019). Recently, Bi et al. 2018 demonstrated high spatial resolution SRS imaging of neurons by tuning the pump and Stoke wavelengths from NIR to the visible region (Bi et al. 2018). Experimentally, they used beta-barium borate crystals to shift the wavelength of both the pump and Stokes to half wavelengths $450 \mathrm{~nm}$ and $520 \mathrm{~nm}$, respectively. The imaging contrast and spatial resolution were significantly improved on using visible wavelength in the SRS technique. Direct imaging ability of visible SRS microscope was demonstrated using unprocessed mouse brain tissue sections. High-resolution SRS images of the brain slice were obtained, covering regions of cortex, subiculum, alveus of the hippocampus, and other areas of neurons and parts of blood vessels (Fig. 10A-D). Strong SRS signals were generated in the 


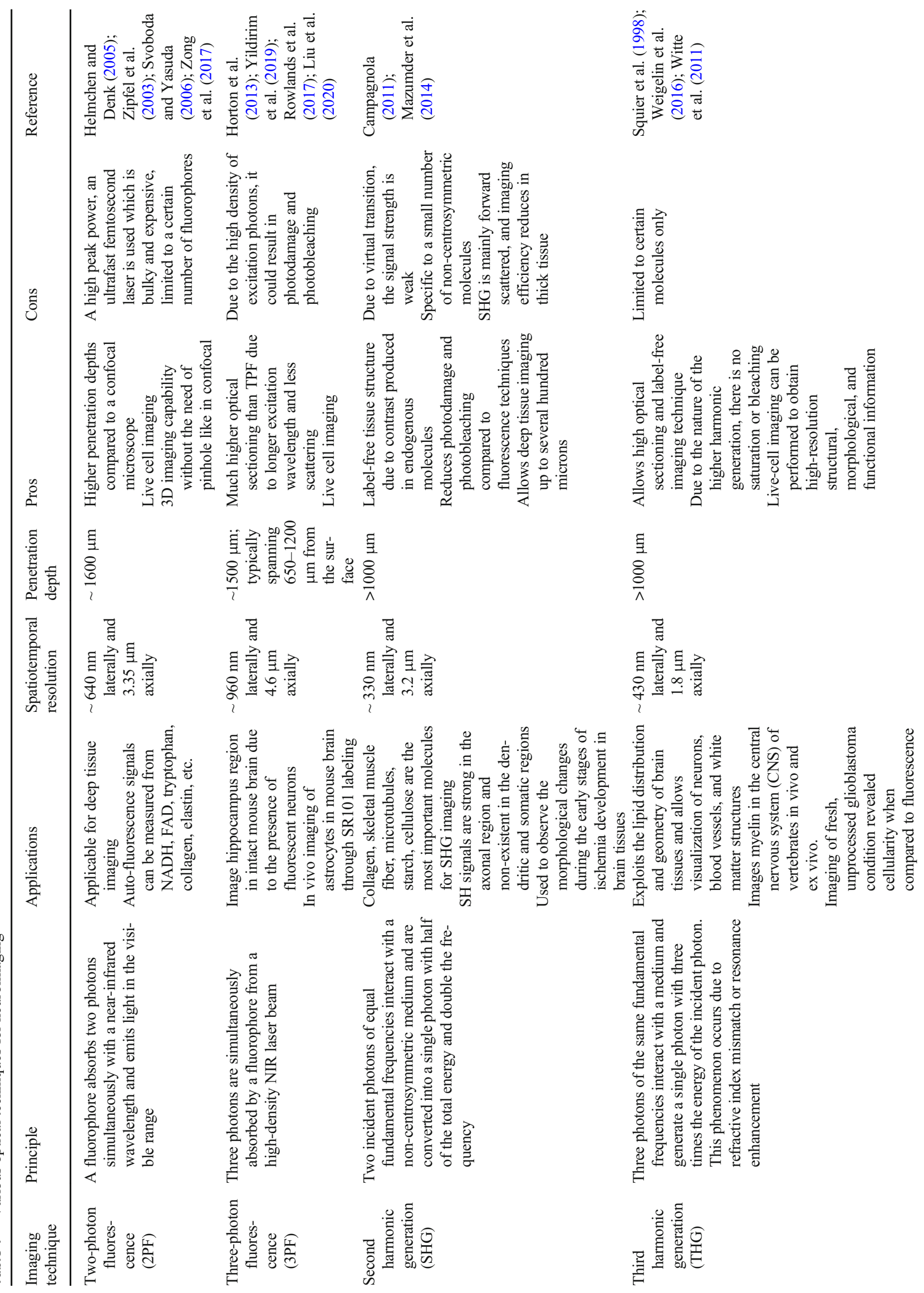




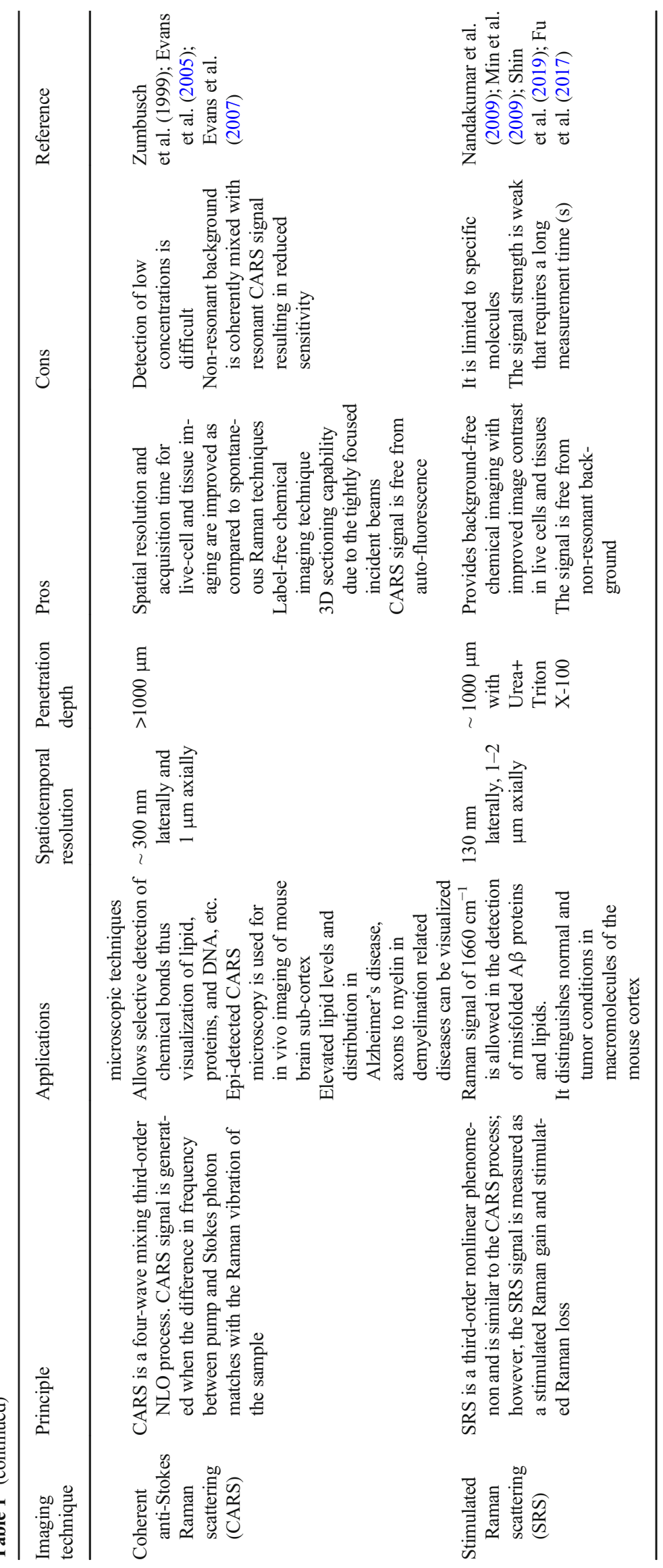


white matter of the brain due to the abundance of lipid content present in the dense myelinated axons found in this region (Fig. 10D). SRS images revealed approximately $1 \mu \mathrm{m}$-diameter, circular enclosed structure on cross-section view and two parallel curves on the longitudinal section view of the axons. The neural network was observed as an extremely crisscrossed pattern of fiber bundles, consisting of highly distributed neurons and blood vessels. Further, visible SRS imaging was able to help distinguish the alveus of the hippocampus from the subiculum regions of the brain. The subiculum region is densely populated with neurons and a scarce amount of bundle fibers. The experiments suggested that visible SRS microscopy can achieve an imaging depth of about $10 \mu \mathrm{m}$, with good image contrast. Likewise, the NIR SRS microscope can substantially improve the penetration depth to approximately $50 \mu \mathrm{m}$ in a similar region.

\section{Conclusion}

The recent developments in advanced optical imaging techniques have generated considerable excitement in brain imaging research. Neuroimaging techniques have widespread applications in the early detection of pathological and physiological changes associated with various neurological disorders. This review provides an overview of various neuroimaging microscopy techniques, with an emphasis on the technical aspects of the methods (Table 1). Further development of these optical imaging modalities will result in an effective alternative to the traditionally employed options, enabling researchers to switch towards an accurate, non-invasive approach to study the neuronal information and enable early diagnosis of neurological disorder. It is also noted that the abovementioned imaging technologies will fortify our understanding of the underlying disease mechanisms and facilitate the early diagnosis of neurological disorders. There are other emerging nonlinear optical techniques such as Brillouin microscopy which have become a tool of interest in the brain research community as it enables label-free, noncontact, and high-resolution imaging of viscoelastic properties of living matter in three dimensions (Mattana et al. 2017; Prevedel et al. 2019).

Acknowledgements We thank Manipal Academy of Higher Education (MAHE), Manipal and Technology Information Forecasting and Assessment Council-Centre of Relevance and Excellence (TIFACCORE) in Pharmacogenomics, Manipal School of Life Sciences, MAHE, for providing the infrastructure and facilities.

Funding Open access funding provided by Manipal Academy of Higher Education, Manipal. We thank the Department of Science and Technology (DST), Government of India, for financial support (Project Number-SERB/MTR/2020/000058, DST/INT/BLG/P-03/2019).

\section{Declarations}

Conflict of interest The authors declare no competing interests.

Open Access This article is licensed under a Creative Commons Attribution 4.0 International License, which permits use, sharing, adaptation, distribution and reproduction in any medium or format, as long as you give appropriate credit to the original author(s) and the source, provide a link to the Creative Commons licence, and indicate if changes were made. The images or other third party material in this article are included in the article's Creative Commons licence, unless indicated otherwise in a credit line to the material. If material is not included in the article's Creative Commons licence and your intended use is not permitted by statutory regulation or exceeds the permitted use, you will need to obtain permission directly from the copyright holder. To view a copy of this licence, visit http://creativecommons.org/licenses/by/4.0/.

\section{References}

Araque A, Martın ED, Perea G, Arellano JI, Buño W (2002) Synaptically released acetylcholine evokes $\mathrm{Ca}^{2+}$ elevations in astrocytes in hippocampal slices. J Neurosci 22(7):2443-2450. https://doi.org/10. 1523/JNEUROSCI.22-07-02443.2002

Bi Y, Yang C, Chen Y, Yan S, Yang G, Wu Y, Zhang G, Wang P (2018) Near-resonance enhanced label-free stimulated Raman scattering microscopy with spatial resolution near $130 \mathrm{~nm}$. Light Sci Appl 7(1):81. https://doi.org/10.1038/s41377-018-0082-1

Brideau C, Poon KW, Colarusso P, Stys PK (2019) Excitation parameters optimized for coherent anti-Stokes Raman scattering imaging of myelinated tissue. J Biomed Opt 24(4):046502. https://doi.org/10. 1117/1.JBO.24.4.046502

Campagnola P (2011) Second harmonic generation imaging microscopy: applications to diseases diagnostics. Anal Chem 83(9):3224-3231. https://doi.org/10.1021/ac1032325

Chen YC, Hsu HC, Lee CM, Sun CK (2015) Third-harmonic generation susceptibility spectroscopy in free fatty acids. J Biomed Opt 20(9): 095013. https://doi.org/10.1117/1.JBO.20.9.095013

Chen Y, Liu S, Liu H, Tong S, Tang H, Zhang C, Yan S, Li H, Yang G, Zhu D, Wang K (2019) Coherent Raman scattering unravelling mechanisms underlying skull optical clearing for through-skull brain imaging. Anal Chem 91(15):9371-9375. https://doi.org/10. 1021/acs.analchem.9b02624

Cheng JX, Jia YK, Zheng G, Xie XS (2002) Laser-scanning coherent anti-Stokes Raman scattering microscopy and applications to cell biology. Biophys J 83(1):502-509. https://doi.org/10.1016/S00063495(02)75186-2

Das S, Chen IC, Rehman KU, Hsu JL, Zhuo GY, Kao FJ (2019a) Background free imaging in stimulated emission fluorescence microscopy. J Opt 21(12):125301. https://doi.org/10.1088/2040-8986/ab4dc2

Das S, Liang YC, Tanaka S, Ozeki Y, Kao FJ (2019b) Synchronized subharmonic modulation in stimulated emission microscopy. Opt Express 27(19):27159-27167. https://doi.org/10.1364/OE.27.027159

Davalos D, Grutzendler J, Yang G, Kim JV, Zuo Y, Jung S, Littman DR, Dustin ML, Gan WB (2005) ATP mediates rapid microglial response to local brain injury in vivo. Nat Neurosci 8(6):752-758. https://doi.org/10.1038/nn1472

Denk W, Strickler JH, Webb WW (1990) Two-photon laser scanning fluorescence microscopy. Science 248(4951):73-76. https://doi. org/10.1126/science.2321027

Dombeck DA, Kasischke KA, Vishwasrao HD, Ingelsson M, Hyman BT, Webb WW (2003) Uniform polarity microtubule assemblies imaged in native brain tissue by second-harmonic generation 
microscopy. Proc Natl Acad Sci U S A 100(12):7081-7086. https:// doi.org/10.1073/pnas.0731953100

Evans CL, Potma EO, Puoris' haag M, Côté D, Lin CP, Xie XS (2005) Chemical imaging of tissue in vivo with video-rate coherent antiStokes Raman scattering microscopy. Proc Natl Acad Sci U S A 102(46):16807-16812. https://doi.org/10.1073/pnas.0508282102

Evans CL, Xu X, Kesari S, Xie XS, Wong ST, Young GS (2007) Chemically-selective imaging of brain structures with CARS microscopy. Opt Express 15(19):12076-12087. https://doi.org/10. 1364/OE.15.012076

Farrar MJ, Wise FW, Fetcho JR, Schaffer CB (2011) In vivo imaging of myelin in the vertebrate central nervous system using third harmonic generation microscopy. Biophys J 100(5):1362-1371. https://doi. org/10.1016/j.bpj.2011.01.031

Fu Y, Huff TB, Wang HW, Wang H, Cheng JX (2008) Ex vivo and in vivo imaging of myelin fibers in mouse brain by coherent antiStokes Raman scattering microscopy. Opt Express 16(24):1939619409. https://doi.org/10.1364/OE.16.019396

Fu D, Yang W, Xie XS (2017) Label-free imaging of neurotransmitter acetylcholine at neuromuscular junctions with stimulated Raman scattering. J Am Chem Soc 139(2):583-586. https://doi.org/10. 1021/jacs.6b10727

Gualda EJ, Filippidis G, Mari M, Voglis G, Vlachos M, Fotakis C, Tavernarakis $N$ (2008) In vivo imaging of neurodegeneration in Caenorhabditis elegans by third harmonic generation microscopy. J Microsc 232(2):270-275. https://doi.org/10.1111/j.1365-2818. 2008.02098.x

Hamada K, Fujita K, Smith NI, Kobayashi M, Inouye Y, Kawata S (2008) Raman microscopy for dynamic molecular imaging of living cells. J Biomed Opt 13(4):044027. https://doi.org/10.1117/1.2952192

Hell SW, Wichmann J (1994) Breaking the diffraction resolution limit by stimulated emission: stimulated-emission-depletion fluorescence microscopy. Opt Lett 19(11):780-782. https://doi.org/10.1364/OL.19.000780

Helmchen F, Denk W (2005) Deep tissue two-photon microscopy. Nat Methods 2(12):932-940. https://doi.org/10.1038/nmeth818

Heo CH, Sarkar AR, Baik SH, Jung TS, Kim JJ, Kang H, Mook-Jung I, Kim HM (2016) A quadrupolar two-photon fluorescent probe for in vivo imaging of amyloid- $\beta$ plaques. Chem Sci 7(7):4600-4646. https://doi.org/10.1039/C6SC00355A

Horton NG, Wang K, Kobat D, Clark CG, Wise FW, Schaffer CB, Xu C (2013) In vivo three-photon microscopy of subcortical structures within an intact mouse brain. Nat Photonics 7(3):205-209. https:// doi.org/10.1038/nphoton.2012.336

Huff TB, Shi Y, Fu Y, Wang H, Cheng JX (2008) Multimodal nonlinear optical microscopy and applications to central nervous system imaging. IEEE J Sel Top Quantum Electron 14(1):4-9. https://doi.org/ 10.1109/JSTQE.2007.913419

Huland DM, Charan K, Ouzounov DG, Jones JS, Nishimura N, Xu C (2013) Three-photon excited fluorescence imaging of unstained tissue using a GRIN lens endoscope. Biomed Opt Express 4(5):652658. https://doi.org/10.1364/BOE.4.000652

Imitola J, Rasmussen S, Liu Y, Chitnis T, Khoury S, Côté D, Xie XS, Lin CP, Sidman RL (2011) Multimodal coherent anti-Stokes Raman scattering microscopy reveals microglia-associated myelin and axonal dysfunction in multiple sclerosis-like lesions in mice. J Biomed Opt 16(2):021109. https://doi.org/10.1117/1.3533312

Ji M, Orringer DA, Freudiger CW, Ramkissoon S, Liu X, Lau D, Golby AJ, Norton I, Hayashi M, Agar NY, Young GS (2013) Rapid, labelfree detection of brain tumors with stimulated Raman scattering microscopy. Sci Transl Med 5(201):201ra119-201ra119. https:// doi.org/10.1126/scitranslmed.3005954

Ji M, Arbel M, Zhang L, Freudiger CW, Hou SS, Lin D, Yang X, Bacskai BJ, Xie XS (2018) Label-free imaging of amyloid plaques in Alzheimer's disease with stimulated Raman scattering microscopy. Sci Adv 4(11):eaat7715. https://doi.org/10.1126/sciadv.aat7715
Johansson PK, Koelsch P (2017) Label-free imaging of amyloids using the intrinsic linear and nonlinear optical properties. Biomed Opt Express 8(2):743-756. https://doi.org/10.1364/BOE.8.000743

Kawakami R, Sawada K, Kusama Y, Fang YC, Kanazawa S, Kozawa Y, Sato S, Yokoyama H, Nemoto T (2015) In vivo two-photon imaging of mouse hippocampal neurons in dentate gyrus using a light source based on a high-peak power gain-switched laser diode. Biomed Opt Express 6(3):891-901. https://doi.org/10.1364/BOE.6.000891

Kiskis J, Fink H, Nyberg L, Thyr J, Li JY, Enejder A (2015) Plaque-associated lipids in Alzheimer's disease brain tissue visualized by nonlinear microscopy. Sci Rep 5(1):13489. https://doi.org/10.1038/srep13489

Klein K, Gigler AM, Aschenbrenner T, Monetti R, Bunk W, Jamitzky F, Morfill G, Stark RW, Schlegel J (2012) Label-free live-cell imaging with confocal Raman microscopy. Biophys J 102(2):360-368. https://doi.org/10.1016/j.bpj.2011.12.027

Kwan AC, Duff K, Gouras GK, Webb WW (2009) Optical visualization of Alzheimer's pathology via multiphoton excited intrinsic fluorescence and second harmonic generation. Opt Express 17(5):36793689. https://doi.org/10.1364/OE.17.003679

Lassonde M, Candel S, Hacker J, Quadrio-Curzio A, Onishi T, Ramakrishnan V, McNutt M (2017) The challenge of neurodegenerative diseases in an aging population. Trends Sci 22:92-93

Le TT, Yue S, Cheng JX (2010) Shedding new light on lipid biology with coherent anti-Stokes Raman scattering microscopy. J Lipid Res 51(11):3091-3102. https://doi.org/10.1194/jlr.R008730

Lee JH, Kim DH, Song WK, Oh MK, Ko DK (2015) Label-free imaging and quantitative chemical analysis of Alzheimer's disease brain samples with multimodal multiphoton nonlinear optical microspectroscopy. J Biomed Opt 20(5):056013. https://doi.org/ 10.1117/1.JBO.20.5.056013

Lim H, Sharoukhov D, Kassim I, Zhang Y, Salzer JL, Melendez-Vasquez CV (2014) Label-free imaging of Schwann cell myelination by third harmonic generation microscopy. Proc Natl Acad Sci U S A 111(50):18025-18030. https://doi.org/10.1073/pnas.1417820111

Liu H, Wang J, Zhuang Z, He J, Wen W, Qiu P, Wang K (2019) Visualizing astrocytes in the deep mouse brain in vivo. J Biophotonics 12(7):e201800420. https://doi.org/10.1002/jbio. 201800420

Liu CJ, Roy A, Simons AA, Farinella DM, Kara P (2020) Three-photon imaging of synthetic dyes in deep layers of the neocortex. Sci Rep 10(1):1-12. https://doi.org/10.1038/s41598-020-73438-w

Lu J, Li C, Singh-Alvarado J, Zhou ZC, Fröhlich F, Mooney R, Wang F (2018) MIN1PIPE: a miniscope 1-photon-based calcium imaging signal extraction pipeline. Cell Rep 23(12):3673-3684. https://doi. org/10.1016/j.celrep.2018.05.062

Matsumoto N, Inoue T, Matsumoto A, Okazaki S (2015) Correction of depth-induced spherical aberration for deep observation using twophoton excitation fluorescence microscopy with spatial light modulator. Biomed Opt Express 6(7):2575-2587. https://doi.org/10.1364/ BOE.6.002575

Mattana S, Caponi S, Tamagnini F, Fioretto D, Palombo F (2017) Viscoelasticity of amyloid plaques in transgenic mouse brain studied by Brillouin microspectroscopy and correlative Raman analysis. J Innov Opt Health Sci 10(06):1742001. https://doi.org/10.1142/ S1793545817420019

Mazumder N, Qiu J, Foreman MR, Romero CM, Hu CW, Tsai HR, Török P, Kao FJ (2012) Polarization-resolved second harmonic generation microscopy with a four-channel Stokes-polarimeter. Opt Express 20(13):14090-14099. https://doi.org/10.1364/OE.20.014090

Mazumder N, Lyn RK, Singaravelu R, Ridsdale A, Moffatt DJ, Hu CW, Tsai HR, McLauchlan J, Stolow A, Kao FJ, Pezacki JP (2013) Fluorescence lifetime imaging of alterations to cellular metabolism by domain 2 of the hepatitis C virus core protein. PLoS One 8(6): e66738. https://doi.org/10.1371/journal.pone.0066738

Mazumder N, Hu CW, Qiu J, Foreman MR, Romero CM, Török P, Kao FJ (2014) Revealing molecular structure and orientation with Stokes 
vector resolved second harmonic generation microscopy. Methods 66(2):237-245. https://doi.org/10.1016/j.ymeth.2013.07.019

Min W, Lu S, Chong S, Roy R, Holtom GR, Xie XS (2009) Imaging chromophores with undetectable fluorescence by stimulated emission microscopy. Nature 461(7267):1105-1109. https://doi.org/10. 1038/nature08438

Mittmann W, Wallace DJ, Czubayko U, Herb JT, Schaefer AT, Looger LL, Denk W, Kerr JN (2011) Two-photon calcium imaging of evoked activity from L5 somatosensory neurons in vivo. Nat Neurosci 14(8):1089-1093. https://doi.org/10.1038/nn.2879

Moree B, Yin G, Lázaro DF, Munari F, Strohäker T, Giller K, Becker S, Outeiro TF, Zweckstetter M, Salafsky J (2015) Small molecules detected by second-harmonic generation modulate the conformation of monomeric $\alpha$-synuclein and reduce its aggregation in cells. J Biol Chem 290(46):27582-27593. https://doi.org/10.1074/jbc.M114. 636027

Nandakumar P, Kovalev A, Volkmer A (2009) Vibrational imaging based on stimulated Raman scattering microscopy. New J Phys 11(3):033026. https://doi.org/10.1088/1367-2630/11/3/033026

Ogawa M, Harada Y, Yamaoka Y, Fujita K, Yaku H, Takamatsu T (2009) Label-free biochemical imaging of heart tissue with high-speed spontaneous Raman microscopy. Biochem Biophys Res Commun 382(2): 370-374. https://doi.org/10.1016/j.bbrc.2009.03.028

Ouzounov DG, Wang T, Wu C, Xu C (2019) GCaMP6 $\Delta$ F/F dependence on the excitation wavelength in 3-photon and 2-photon microscopy of mouse brain activity. Biomed Opt Express 10(7):3343-3352. https://doi.org/10.1364/BOE.10.003343

Perea G, Araque A (2005) Properties of synaptically evoked astrocyte calcium signal reveal synaptic information processing by astrocytes. J Neurosci 25(9):2192-2203. https://doi.org/10.1523/JNEUROSCI. 3965-04.2005

Pittolo S, Lee H, Lladó A, Tosi S, Bosch M, Bardia L, Gómez-Santacana X, Llebaria A, Soriano E, Colombelli J, Poskanzer KE (2019) Reversible silencing of endogenous receptors in intact brain tissue using 2-photon pharmacology. Proc Natl Acad Sci U S A 116(27): 13680-13689. https://doi.org/10.1073/pnas.1900430116

Poulon F, Pallud J, Varlet P, Zanello M, Chretien F, Dezamis E, AbiLahoud G, Nataf F, Turak B, Devaux B, Abi Haidar D (2018) Realtime brain tumor imaging with endogenous fluorophores: a diagnosis proof-of-concept study on fresh human samples. Sci Rep 8(1): 14888. https://doi.org/10.1038/s41598-018-33134-2

Prevedel R, Diz-Muñoz A, Ruocco G, Antonacci G (2019) Brillouin microscopy: an emerging tool for mechanobiology. Nat Methods 16(10):969-977. https://doi.org/10.1038/s41592-019-0543-3

Psilodimitrakopoulos S, Petegnief V, de Vera N, Hernandez O, Artigas D, Planas AM, Loza-Alvarez P (2013) Quantitative imaging of microtubule alteration as an early marker of axonal degeneration after ischemia in neurons. Biophys J 104(5):968-975. https://doi.org/10. 1016/j.bpj.2013.01.020

Redlich MJ, Lim H (2019) A method to measure myeloarchitecture of the murine cerebral cortex in vivo and ex vivo by intrinsic thirdharmonic generation. Front Neuroanat 13:65. https://doi.org/10. 3389/fnana.2019.00065

Rodríguez C, Liang Y, Lu R, Ji N (2018) Three-photon fluorescence microscopy with an axially elongated Bessel focus. Opt Lett 43(8): 1914-1917. https://doi.org/10.1364/OL.43.001914

Ross CA, Poirier MA (2004) Protein aggregation and neurodegenerative disease. Nat Med 10(7):S10-S17. https://doi.org/10.1038/nm1066

Rowlands CJ, Park D, Bruns OT, Piatkevich KD, Fukumura D, Jain RK, Bawendi MG, Boyden ES, So PT (2017) Wide-field three-photon excitation in biological samples. Light Sci Appl 6(5):e16255. https:// doi.org/10.1038/lsa.2016.255
Sacconi L, Dombeck DA, Webb WW (2006) Overcoming photodamage in second-harmonic generation microscopy: real-time optical recording of neuronal action potentials. Proc Natl Acad Sci U S A 103(9):3124-3129. https://doi.org/10.1073/pnas.0511338103

Sahu P, Mazumder N (2020) Advances in adaptive optics-based twophoton fluorescence microscopy for brain imaging. Lasers Med Sci 35(2):317-328. https://doi.org/10.1007/s10103-019-02908-z

Shin KS, Francis AT, Hill AH, Laohajaratsang M, Cimino PJ, Latimer CS, Gonzalez-Cuyar LF, Sekhar LN, Juric-Sekhar G, Fu D (2019) Intraoperative assessment of skull base tumors using stimulated Raman scattering microscopy. Sci Rep 9(1):20392. https://doi.org/ 10.1038/s41598-019-56932-8

Sinefeld D, Paudel HP, Ouzounov DG, Bifano TG, Xu C (2015) Adaptive optics in three-photon fluorescence microscopy. CLEO: OSA Technical Digest (online) STu2K.8. https://oi.org/10.1364/ CLEO SI.2015.STu2K.8

Sivaguru M, Durgam S, Ambekar R, Luedtke D, Fried G, Stewart A, Toussaint KC (2010) Quantitative analysis of collagen fiber organization in injured tendons using Fourier transform-second harmonic generation imaging. Opt Express 18(24):24983-24993. https://doi. org/10.1364/OE.18.024983

Soto C (2003) Unfolding the role of protein misfolding in neurodegenerative diseases. Nat Rev Neurosci 4(1):49-60. https://doi.org/10. 1038/nrn1007

Squier JA, Müller M, Brakenhoff GJ, Wilson KR (1998) Third harmonic generation microscopy. Opt Express 3(9):315-324. https://doi.org/ 10.1364/OE.3.000315

St. Croix CM, Shand SH, Watkins SC (2005) Confocal microscopy: comparisons, applications, and problems. Biotechniques 39(6):S2S5. https://doi.org/10.2144/000112089

Sun CK, Liu WM, Liao YH (2019) Study on melanin enhanced third harmonic generation in a live cell model. Biomed Opt Express 10(11):5716-5723. https://doi.org/10.1364/BOE.10.005716

Svoboda K, Yasuda R (2006) Principles of two-photon excitation microscopy and its applications to neuroscience. Neuron 50(6):823-839. https://doi.org/10.1016/j.neuron.2006.05.019

Tilbury K, Campagnola PJ (2015) Applications of second-harmonic generation imaging microscopy in ovarian and breast cancer. Perspect Medicin Chem 7:PMC-S13214. 10.4137\%2FPMC.S13214

Weigelin B, Bakker GJ, Friedl P (2016) Third harmonic generation microscopy of cells and tissue organization. J Cell Sci 129(2):245-255. https://doi.org/10.1242/jcs. 152272

Weller J, Budson A (2018) Current understanding of Alzheimer's disease diagnosis and treatment. F1000Res 7:F1000 Faculty Rev-1161. https://dx.doi.org/10.12688\%2Ff1000research.14506.1

Witte S, Negrean A, Lodder JC, De Kock CP, Silva GT, Mansvelder HD (2011) Label-free live brain imaging and targeted patching with third-harmonic generation microscopy. Proc Natl Acad Sci U S A 108(15):5970-5975. https://doi.org/10.1073/pnas.1018743108

Wokosin DL, Centonze VE, Crittenden S, White J (1996) Three-photon excitation fluorescence imaging of biological specimens using an all-solid-state laser. Bioimaging 4(3):208-214. https://doi.org/10. 1002/1361-6374(199609)4:3\%3C208::AID-BIO11\%3E3.0.CO;2-J

Xu C, Zipfel W, Shear JB, Williams RM, Webb WW (1996) Multiphoton fluorescence excitation: new spectral windows for biological nonlinear microscopy. Proc Natl Acad Sci U S A 93(20):10763-10768. https://doi.org/10.1073/pnas.93.20.10763

Yildirim M, Sugihara H, So PT, Sur M (2019) Functional imaging of visual cortical layers and subplate in awake mice with optimized three-photon microscopy. Nat Commun 10(1):177. https://doi.org/ 10.1038/s41467-018-08179-6 
Zhang XY, Yang ZL, Lu GM, Yang GF, Zhang LJ (2017) PET/MR imaging: new frontier in Alzheimer's disease and other dementias. Front Mol Neurosci 10:343. https://doi.org/10.3389/fnmol.2017. 00343

Zipfel WR, Williams RM, Christie R, Nikitin AY, Hyman BT, Webb WW (2003) Live tissue intrinsic emission microscopy using multiphoton-excited native fluorescence and second harmonic generation. Proc Natl Acad Sci U S A 100(12):7075-7080. https://doi. org/10.1073/pnas.0832308100

Zong W, Wu R, Li M, Hu Y, Li Y, Li J, Rong H, Wu H, Xu Y, Lu Y, Jia $\mathrm{H}$ (2017) Fast high-resolution miniature two-photon microscopy for brain imaging in freely behaving mice. Nat Methods 14(7):713-719. https://doi.org/10.1038/nmeth.4305

Zumbusch A, Holtom GR, Xie XS (1999) Three-dimensional vibrational imaging by coherent anti-Stokes Raman scattering. Phys Rev Lett 82(20):4142. https://doi.org/10.1103/PhysRevLett.82.4142

Publisher's note Springer Nature remains neutral with regard to jurisdictional claims in published maps and institutional affiliations. 